\title{
Sub-Grain Scale Digital Image Correlation by Electron Microscopy for Polycrystalline Materials during Elastic and Plastic Deformation
}

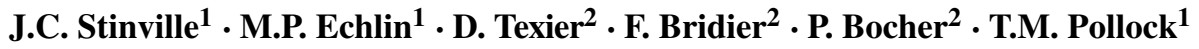

\begin{abstract}
Damage during loading of polycrystalline metallic alloys is localized at or below the scale of individual grains. Quantitative assessment of the heterogeneous strain fields at the grain scale is necessary to understand the relationship between microstructure and elastic and plastic deformation. In the present study, digital image correlation (DIC) is used to measure the strains at the sub-grain level in a polycrystalline nickel-base superalloy where plasticity is localized into physical slip bands. Parameters to minimize noise given a set speckle pattern (introduced by chemical etching) when performing DIC in a scanning electron microscope (SEM) were adapted for measurements in both plastic and elastic regimes. A methodology for the optimization of the SEM and DIC parameters necessary for the minimization of the variability in strain measurements at high spatial resolutions is presented. The implications for detecting the early stages of damage development are discussed.
\end{abstract}

Keywords High resolution digital image correlation. DIC $\cdot$ Scanning electron microscopy $\cdot$ SEM $\cdot$ Slip bands . Polycrystalline materials $\cdot$ In-situ tensile testing $\cdot$ René 88DT $\cdot$ Strain localization

M.P. Echlin

mechlin@engineering.ucsb.edu

J.C. Stinville

stinville@engineering.ucsb.edu

1 University of California Santa Barbara, Santa Barbara, USA

2 Ecole de Technologie Superieure, Montreal, Canada

\section{Introduction}

Damage during cyclic loading of polycrystalline metallic alloys involves accumulation of plastic strain at the scale of individual grains, particularly in the vicinity of grain boundaries and interfaces. For example, Miao et al. [1,2] reported for the nickel-based alloy René 88DT under very high cycle fatigue loading that cracks initiated in high Schmid factor grains parallel to and slightly offset from coherent twin boundaries. To develop predictive models for monotonic and cyclic loading, there is a need for quantitative assessment of the heterogeneous strain fields at the microscopic scale, to better relate the local mechanical behavior to the global loading conditions. Crystal plasticity simulations based upon either molecular dynamics or finite elements are currently being developed to address these microstructuralinfluenced phenomena $[3,4]$ and localization of plasticity along slip bands or persistent slip bands [5]. Experimental data on strain localization via in situ SEM DIC is available [6-14] at the microscale, however few experiments [1517] have resolved subgrain strain fields at the scale of the localization of plasticity (slip bands).

The combination of scanning electron microscopy (SEM) and digital image correlation (DIC) has recently emerged as a robust method for experimental quantification of 2-D in-plane strain fields at the microstructure scale $[6,7,12,13,15,17-19]$. However, it is still challenging to obtain the necessary spatial resolution to measure the strain field at the micron and submicron scale, especially when plasticity (slip) is involved. In several studies [6, 13, 17], grain-scale spatial resolution has been achieved in materials with $\mathrm{mm}$ - or $\mathrm{cm}$-scale grains. However, since many high strength structural materials have grain sizes of $100 \mu \mathrm{m}$ or 
smaller, it is necessary to refine the DIC measurements to finer scales. Tatschl et al. [20] succeeded in measuring strain heterogeneities by DIC using submicron scale speckle on grains with an average size of about $100 \mu \mathrm{m}$. Moreover, they demonstrate the combination of DIC measurement and electron backscatter diffraction (EBSD) measurements in order to relate crystallographic data with strain fields that develop during tensile loading. While heterogeneities were detected at the grain-scale, the spatial resolution was insufficient to quantify strains induced by localized slip within grains. To gain insights to the role of interfaces such as grain and twin boundaries, higher resolution observations are required, especially when plasticity is involved. The use of SEM imaging for DIC comes at the cost of complex image distortions $[15,18,21,22]$ and sample charging related beam drift. Kammers and Daly [15, 18] suggest SEM parameters to reduce these distortions and also present electron beam drift correction routines. In this paper, DIC measurements are applied to a nickel based superalloy with an average grain size of $26 \mu \mathrm{m}$ to give insight into straining processes at the sub-grain scale, where plastic strain localization was directly correlated with physical slip bands. Given a set field of view and speckle pattern, scanning electron microscope parameters are presented that significantly reduce the beam distortion errors inherent to SEM imaging without using complex drift correction routines. Additionally, DIC parameters are adapted to permit the study of polycrystalline materials under elastic and plastic deformation conditions.

\section{Material and Experimental Procedure}

\section{Material}

A commercial polycrystalline powder metallurgy processed nickel-based superalloy, René 88DT, was investigated in this research. The nominal composition of this alloy is $13 \% \mathrm{Co}, 16 \% \mathrm{Cr}, 4 \% \mathrm{Mo}, 4 \% \mathrm{~W}, 2.1 \% \mathrm{Al}, 3.7 \% \mathrm{Ti}, 0.7$ $\% \mathrm{Nb}, 0.03 \% \mathrm{C}, 0.015 \% \mathrm{~B}$ (wt \%) [23]. The microstructure of the alloy consists of a $\gamma$ matrix and two populations of gamma prime $\left(\gamma^{\prime}\right)$ precipitates: larger secondary and nmscale tertiary $\gamma /$ within the $\gamma$ grains [23]. The size of the secondary $\gamma^{\prime}$ phase is about $100-200 \mathrm{~nm}$, while tertiary $\gamma^{\prime}$ precipitates are several nanometers in diameter. Crystallographic features have been previously studied using EBSD measurements [24] showing that the material possesses very weak crystallographic texture, a large population of $\Sigma 3$ boundaries (58\% of the total boundary fraction), an average grain size of $26 \mu \mathrm{m}$, and a low fraction of large grains on the order of two to five times of the average grain size.

\section{Scanning Electron Microscopy and Mechanical Testing}

Tensile tests were performed at room temperature in-situ in a FEI Helios FEG system (FIB-SEM) on flat dogboneshaped specimens with a gauge cross-section of $1 \times 3 \mathrm{~mm}$ and a gauge length of $15 \mathrm{~mm}$ at quasti-static loading conditions with initial strain rates between $10^{-3}$ to $10^{-4}$. Specimens were ground with $\mathrm{SiC}$ papers and chemicalmechanically polished with $0.05 \mu \mathrm{m}$ colloidal silica for 12 hours to remove any residual surface deformation. Prior to deformation, secondary electron images and EBSD maps were acquired. The EBSD maps were taken with an EDAX Hikari XP detector with a step size of $1 \mu \mathrm{m}$. Interrupted tensile tests were performed on the René 88DT samples in the elastic and plastic regimes, at strain levels of $0.29 \%$ and $0.98 \%$, respectively. Strain was measured in-situ using fiducial markers located at both ends of the gauge length. The macroscopic tensile stress-strain curve is shown in Fig. 1 and the values of the macroscopic strain for the two investigated levels are reported. The axial loading direction will be referred to as the $\mathrm{x}$-direction and vertically oriented in all strain maps. In order to minimize distortion errors inherent to SEM imaging [15, 18], SEM parameters were chosen following the guidelines of Kammers and Daly [15, 18]. High magnification images were taken at horizontal field widths (HFWs) of $85 \mu \mathrm{m}$ to reduce distortions. Large electron beam spot sizes (5) and large dwell times ( $20 \mu \mathrm{s}, 6$ minutes per image), were used to reduce drift distortions $[15,18]$. In addition, low acceleration voltages of $5 \mathrm{kV}$ were used to minimize charging effects and reduce drift distortion.

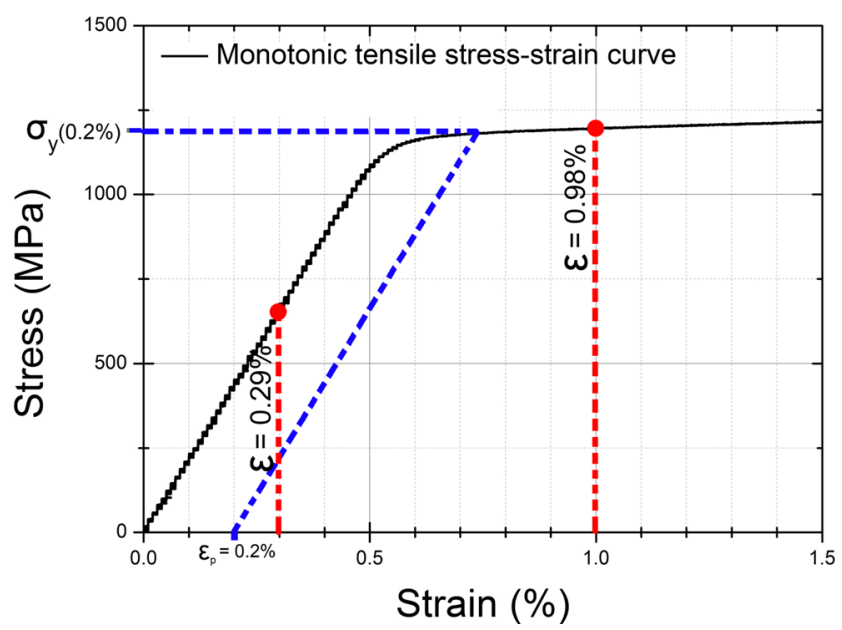

Fig. 1 Macroscopic tensile stress-strain curve for the René 88DT alloy. The values of the macroscopic strain for the two investigated strain levels are reported using red dashed lines. The $0.2 \%$ yield stress is denoted using a dashed blue line 
Short working distances of $5 \mathrm{~mm}$ were used to obtain higher spatial imaging resolution and electron beam stability.

\section{DIC Pattern}

The speckle pattern used for DIC was formed by preferentially etching the intrinsic microstructural features of the René 88DT alloy. Chemical-mechanically polishing with $0.05 \mu \mathrm{m}$ colloidal silica for 12 hours revealed the secondary and tertiary $\gamma^{\prime}$ precipitates in this alloy. Additionally, specimens were heated at $350{ }^{\circ} \mathrm{C}$ for several minutes in order to increase the contrast between the secondary and tertiary $\gamma^{\prime}$ phase and the $\gamma$ matrix. The speckle pattern is stable both upon straining and at temperatures at least to $650{ }^{\circ} \mathrm{C}$ due to the stability of the $\mathrm{L} 1_{2}$ precipitate microstructure, as shown elsewhere [25]. Figure 2 presents a typical SEM image used for DIC measurements with the corresponding EBSD maps of the same sample area. The speckle pattern is composed of secondary and tertiary circular $\gamma^{\prime}$ precipitates ranging from 10-200 $\mathrm{nm}$ in diameter. The use of the secondary and tertiary $\gamma^{\prime}$ phase as the speckle pattern for DIC avoids problems that may be associated with the adhesion of some deposited patterns as described by Sutton [26], because the $\gamma^{\prime}$ phase is a thermodynamically stable constituent of the René 88DT alloy. The red and black boxes in Fig. 2(c and d) indicate subset sizes of $21 \times 21$ pixels

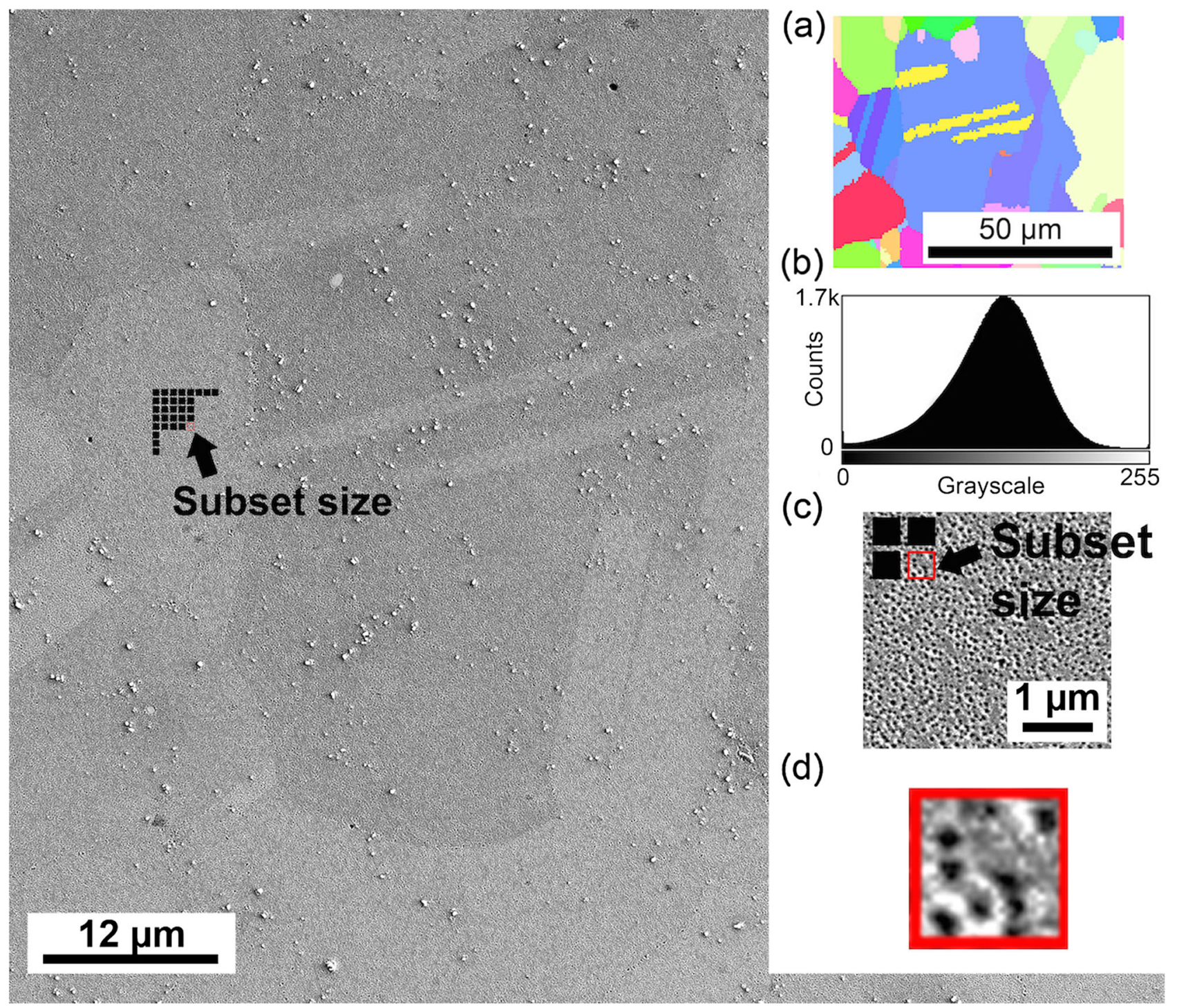

Fig. 2 A SEM micrograph of one region of interest from a DIC experiment, imaged at a magnification of $1500 \times$ with a HFW of $85 \mu \mathrm{m}$. (a) The associated EBSD map of the same region of interest. (b) A grayscale histogram of the image in (a). (c-d) Enlarged images of the speckle pattern contained in a subset region. The black and red boxes indicate a subset size of 21 pixels $(0.4 \mu \mathrm{m})$ 
$(0.4 \times 0.4 \mu \mathrm{m})$ used for DIC measurements. Each subset contains more than $9 \gamma^{\prime}$ precipitate features for each DIC pattern matching. The chemical-mechanical polishing also reveals the microstructure, including the grain and twin boundary locations, allowing for direct spatial registration of the DIC strain maps onto the EBSD maps.

\section{Digital Image Correlation}

The in-plane displacement fields at the microscopic scale were obtained using DIC open source software (OpenDIC) [27] and the commercial software VIC 2D 2009 [28]. The SEM images $(4096 \times 3775$ pixels $)$ were divided into custom sized subsets ranging from $9 \times 9$ to $101 \times 101$ pixels regularly spaced by a step size in both the horizontal and vertical directions. DIC measurements were constructed by comparing images from each deformation step to images of the undeformed specimen.

\section{OpenDIC [27]}

The OpenDIC software uses a correlation that is based on the zero-normalized cross-correlation (ZNCC) criterion [29]. Deformed images were interpolated by a factor of 10 using a biquintic polynomial interpolation algorithm. This interpolation method produces a theoretical accuracy of 0.1 pixel (about $6.2 \mathrm{~nm}$ at $\mathrm{HFW}$ of $85 \mu \mathrm{m}$ ) for the displacements within each subset [29]. A Matlab companion application calculates and plots the in-plane Lagrangian strain fields $\left(\epsilon_{x x}, \epsilon_{y y}\right.$ and $\left.\epsilon_{x y}\right)$ from the displacement fields $U_{X}$ and $U_{Y}$ in the $\mathrm{X}$ (loading) direction and $\mathrm{Y}$ (transverse) directions, respectively. The strain calculation is based on an isoparametric 2-D finite element formalism using subset centers as nodes with four Gauss bilinear interpolation points per element. No smoothing or filtering was applied after the calculation of strain fields using OpenDIC.

\section{Vic 2D 2009 [28]}

The software Vic 2D 2009 was also used to calculate strain fields. The software parameters used include a zero-normalized squared difference correlation criterion for correlation and a $5 \times 5$ subset decay kernel matrix (minimum decay filter available) for deriving strain values [30].

\section{Spatial Distortions}

The use of SEM imaging for DIC comes at the cost of complex image distortions. Therefore, their effects on the strain variability must be evaluated. For instance, spatial distortions were measured by SEM imaging of mesh transmission electron microscopy (TEM) reference grids. SEM images were acquired at different magnifications, ranging from HFW of $1280 \mu \mathrm{m}$ to $128 \mu \mathrm{m}$. Deviations, in pixels, between the known geometry of the TEM grid and SEM images of the grid, shown in Fig. 3, were measured using image analysis software. The maximum deviation was measured using an edge detection algorithm to (a)

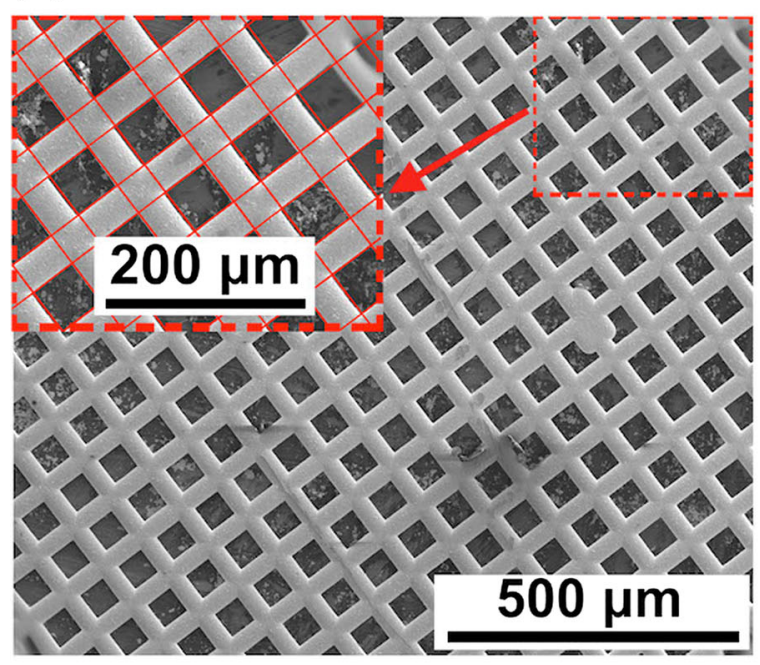

(b)

$\mathrm{HFW}: \mathbf{\square} 1280 \mu \mathrm{m} \bullet 256 \mu \mathrm{m} \wedge 128 \mu \mathrm{m}$

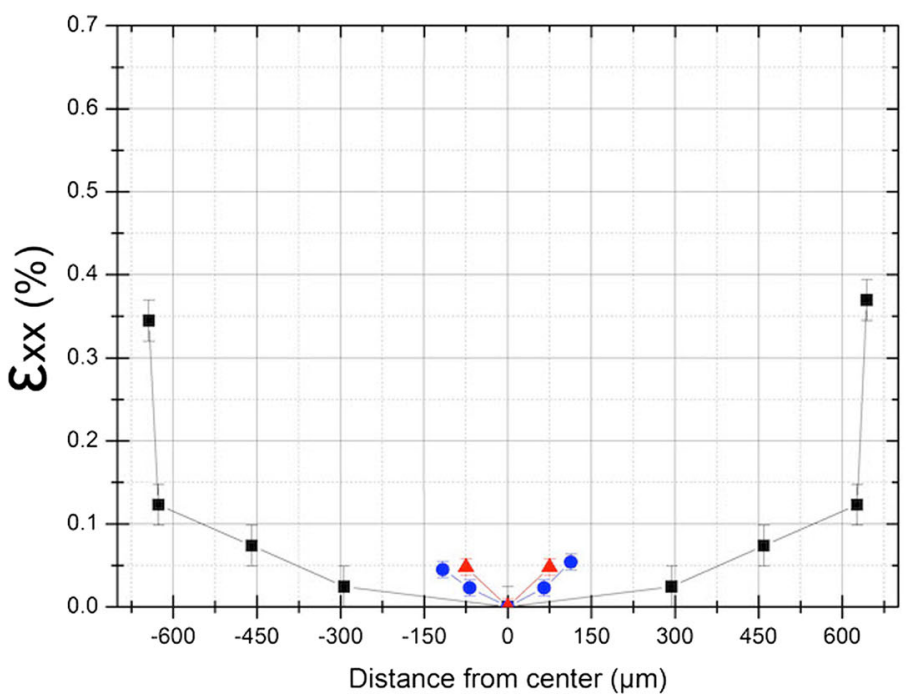

Fig. 3 A mesh TEM grid imaged by SEM, (a), is compared with the known grid geometry shown in the inset image, (a), to measure the displacements and calculate strains (b) for HFWs of 128 to $1280 \mu \mathrm{m}$, which result from spatial distortions. At HFWs of $128 \mu \mathrm{m}$ and below, the spatial distortions result in strain errors below $0.05 \%$. Error bars are calculated from the edge detection location accuracy, which is 1 pixel 

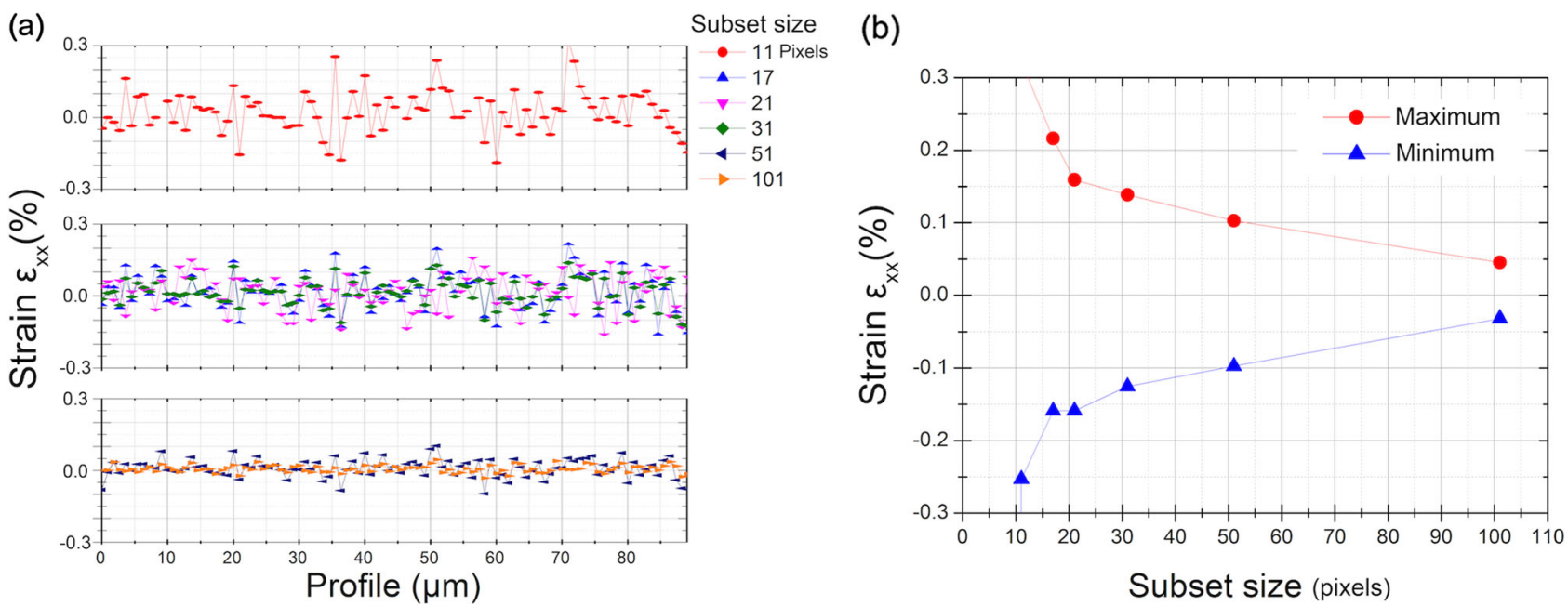

Fig. 4 Variability in DIC strain measurements can be reduced by increasing the subset size, which worsens the spatial resolution. (a) Strain profile measurements were extracted along the image diagonals in DIC measurements calculated using OpenDIC made from image pairs with no applied load for HFWs of $85 \mu \mathrm{m}$ with a step size of 5 pixels, as a function of DIC subset size. The variability in the strain measurement for each subset size, shown in (b), sharply decreases until a subset size of 21 pixels after which it decreases roughly linearly

define the grid location in the SEM images compared with the known grid location, shown in red in Fig. 3(a). Strain was calculated from the deviations in relation to the HFW and result in distortions induced maximum strains of less than $0.05 \%$ for HFW below $256 \mu \mathrm{m}$. Therefore, HFWs at or less than $256 \mu \mathrm{m}$ are used to reduce spatial distortions.

\section{Optimization of Subset Size and HFW for SEM DIC}

High spatial resolution (small subset and step sizes) is necessary in order to study deformation mechanisms at the subgrain scale in polycrystalline materials. However, DIC requires at least several speckle features per subset defined with an optimal number of pixels for correlation [31-33]. Therefore, for a fixed imaging resolution the HFW and the subset size control the minimum variability in displacement measurements. In addition for SEM DIC, distortions are introduced which are dependent upon the HFW and microscope imaging conditions $[15,18]$ and have been characterized as being either spatial or drift related [15, 21, 22, 34]. In the following section, the effect of HFW and subset size are investigated in order to limit variability in the DIC strain measurement.
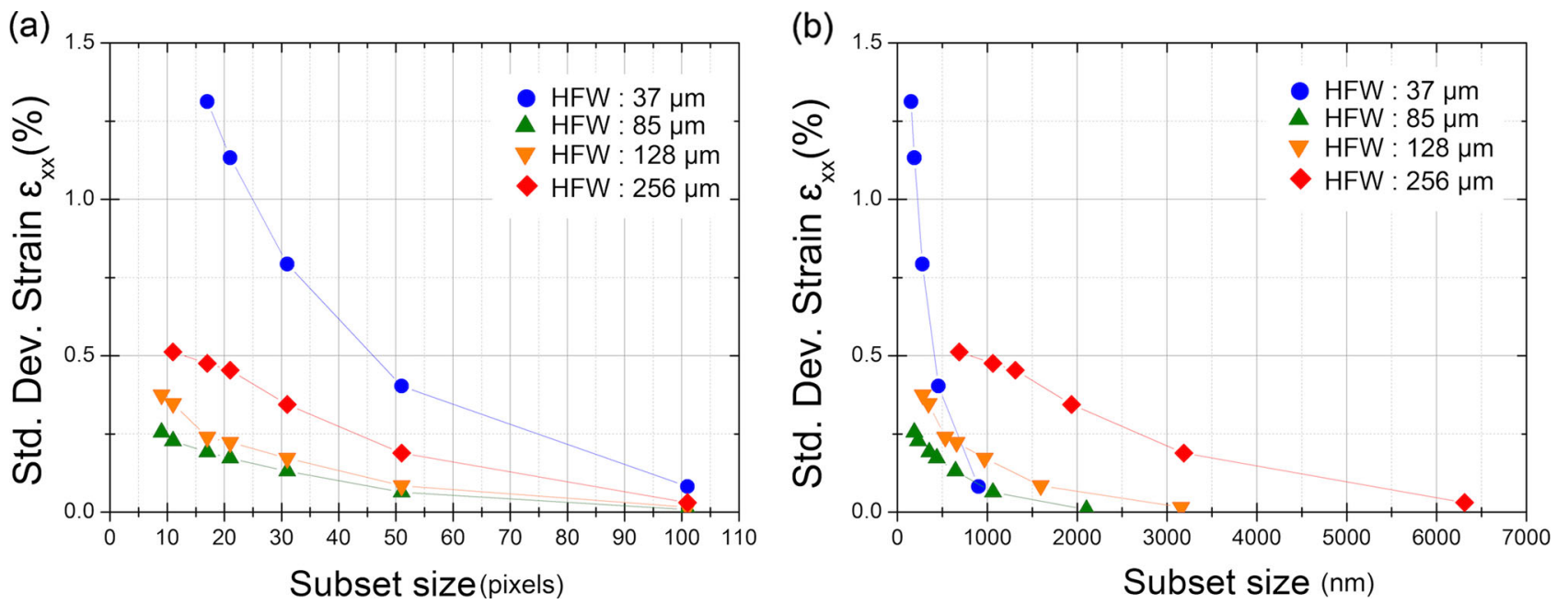

Fig. 5 The variability in DIC strain measurements are plotted for subsets of, (a), 9 to 101 pixels and, (b), 152-6312 nms for HFWs ranging between 37 to $256 \mu \mathrm{m}$ to determine the parameters the give the least strain variability with the smallest subset size and the largest HFW. All calculations were made using a step size of 5 pixels 
(a)

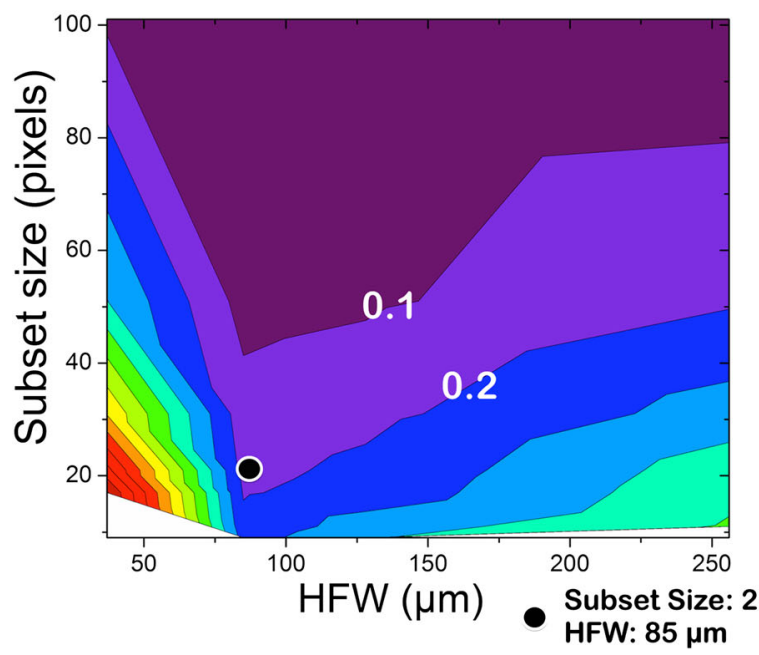

(b)

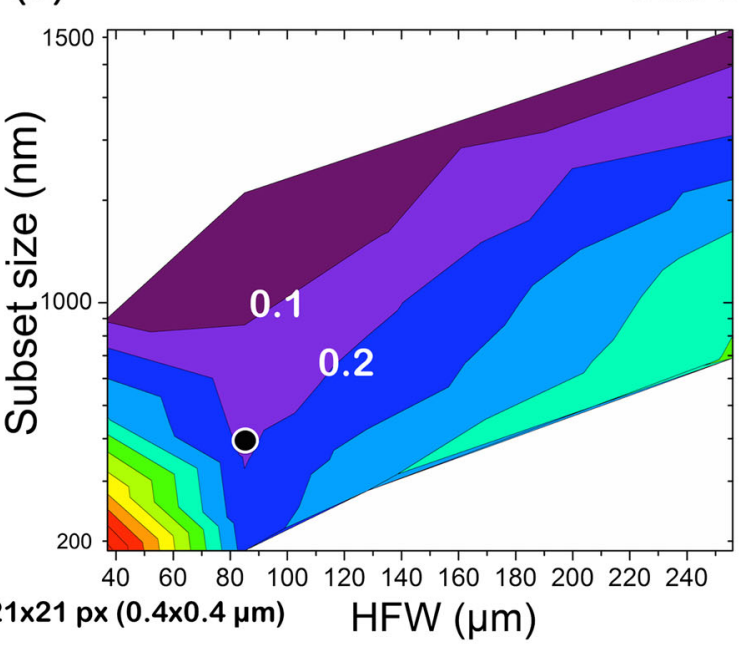

Std. Dev. $\varepsilon_{x x}(\%)$

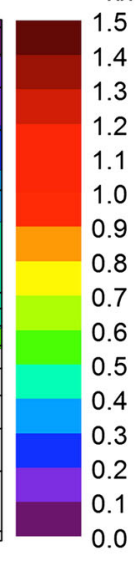

Fig. 6 A contour plot of the standard deviation in strain as a function of subset size, by pixels (a) and nanometers (b), and HFW. The black dots indicate the SEM DIC parameters used to measure strain fields with the largest HFW and highest spatial resolution (small subset size), while minimizing the strain variability

The method described presently can be used to determine the optimal HFW and subset size for high resolution SEM DIC measurements at a fixed speckle pattern and for a given set of microscope parameters. Pairs of images from the same region of interest were collected from unstrained samples at HFWs of 37, 85, 128, and $256 \mu \mathrm{m}$ and strain measurements were made without filtering using the OpenDIC software for subset sizes ranging from 9-101 pixels $(150-6000 \mathrm{~nm})$ at a constant 5 pixel step size. As expected, strain profile measurements along the image diagonal, Fig. 4(a), for each of the subset sizes shows that the variability in the strain measurement decreases with increasing subset size. This trend is summarized in Fig. 4(b) where the variability between the maximum and minimum strain decreases with increasing subset size. Once the relation between subset size and strain variability becomes linear the gains for increasing the subset size diminish. The standard deviation in strain as a function of subset size in pixels, Fig. 5(a), and nanometers, Fig. 5(b), for a range of HFWs show that a $85 \mu \mathrm{m}$ HFW maximizes the imaging area while maintaining the highest spatial resolution and a low strain measurement variability for all subset sizes. A contour plot of the standard deviation in strain measurements as a function of subset size and HFW, Fig. 6, expresses the ideal parameters necessary to increase the spatial resolution (reduce subset size) and collect the largest field of view with strain variability below 0.2 . The optimized parameters used to resolve the strain fields during macroscopic loading conditions with variability less than 0.2 are indicated in Fig. 6, inducing strain errors below $0.15 \%$ as shown in Fig. 4(b). At HFWs larger than $85 \mu \mathrm{m}$, (a) HFW : $414 \mu \mathrm{m}$

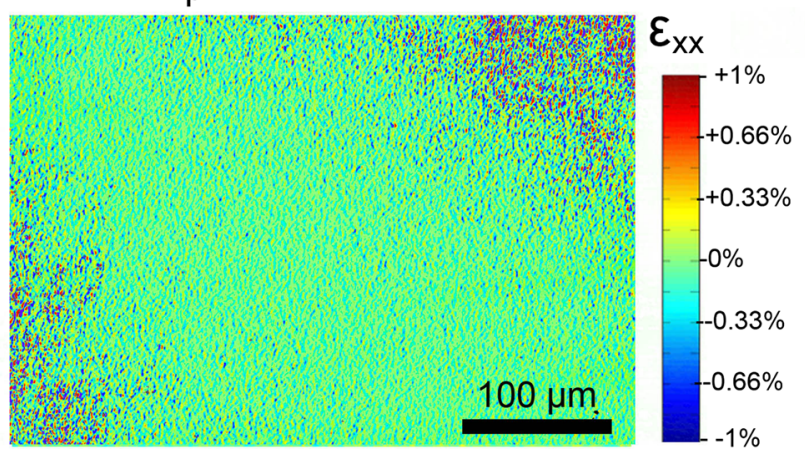

(b) HFW : $207 \mu \mathrm{m}$

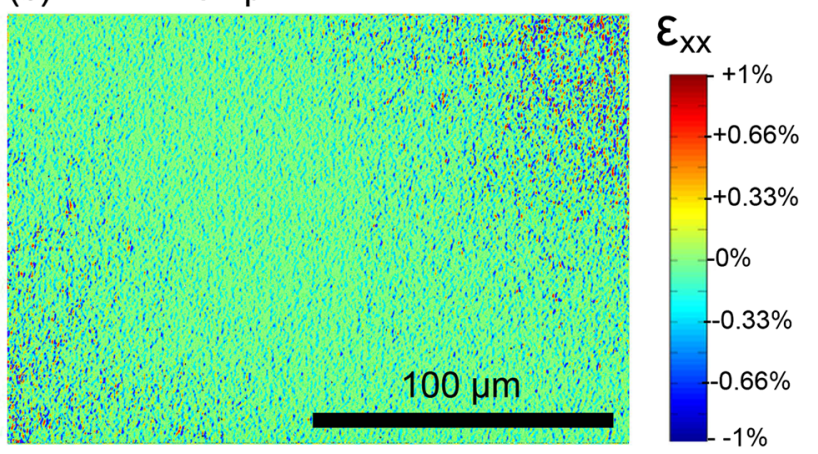

Fig. 7 The strain fields measured from the same region of interest between a pair of images in an unloaded sample at HFWs of $414 \mu \mathrm{m}$ and 207 $\mu \mathrm{m}$ are shown in (a) and (b), respectively. Local strains of up to $1 \%$ are introduced near the edges of the field of view 
for all subset sizes, the variability in strain increases. For example, at HFWs of $414 \mu \mathrm{m}$ and $207 \mu \mathrm{m}$, local strains of up to $1 \%$ are introduced near the edges of the field of view in Fig. 7, which are predominantly due to spatial distortions $[15,21,22,34]$. At HFWs smaller than $85 \mu \mathrm{m}$, for all subset sizes, large variability in strain up to 1.5 standard deviations from the mean are observed. This variability is introduced from SEM imaging distortions, especially those related to drift [15]. Working at small subset size induces higher variability in the strain measurements. However, at an optimal HFW of $85 \mu \mathrm{m}$ the variability remains low for small subset sizes. The presented method makes possible, for a given speckle pattern and a set of microscope parameters, a way to determine the effect of the combined distortions, DIC parameters, and SEM parameters on the variability in strain measurements.

\section{SEM Beam Scanning Defects}

The SEM scan pattern generator for the FEI Helios microscope used in this experiment, is stable when translating the beam along the $\mathrm{X}$ direction, but can experience random stepping errors when incrementing the raster in the $\mathrm{Y}$ direction as shown in Fig. 8(c). Displacement and strain maps calculated from pairs of images from the same region of interest at HFWs of $85 \mu \mathrm{m}$ with a subset size of $21 \times 21$ pixels are presented in Fig. 8(a and c) and Fig. 8(b and d), respectively. The variability in displacement and therefore strain is induced by SEM imaging through distortions and detector noise. Low displacement variations of less than 1 pixel were measured along the horizontal $(\mathrm{X})$ scan direction. Accordingly, the errors induced by the use of SEM imaging in the $\epsilon_{x x}$ field are around $0.15 \%$. The electron beam parameters and an optimum HFW enable high strain resolution in the $\epsilon_{x x}$ field for DIC measurements in the SEM even for small subset sizes. However, Fig. 8(c) shows that there are significant discontinuities in the displacement field along the vertical direction, which induce strain bands in the $\epsilon_{y y}$ field with magnitudes up to $1 \%$. These discontinuities indicate that there are beam scanning defects during image acquisition. In order to avoid displacement errors induced by this scanning defect, two images were taken for each field of view, one with and one without a $90^{\circ}$ scan rotation applied. Displacements are calculated from the scan rotated image that has the horizontal SEM scan direction aligned with the principal stain, $\epsilon_{x x}$ or $\epsilon_{y y}$, being measured. This methodology aligns the stable beam scan direction, horizontal for the SEM used, with the component of the strain (a)

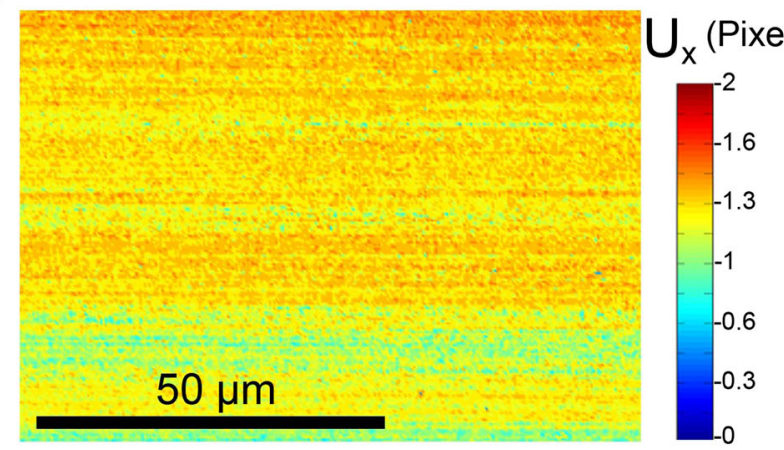

(c)

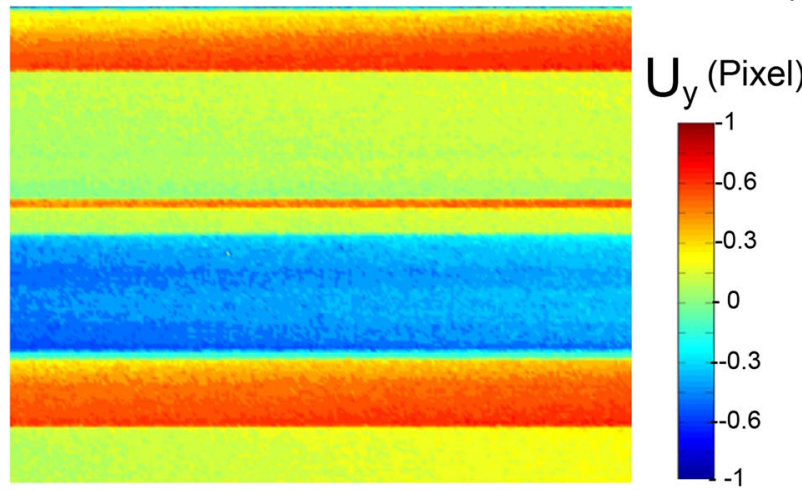

(b)

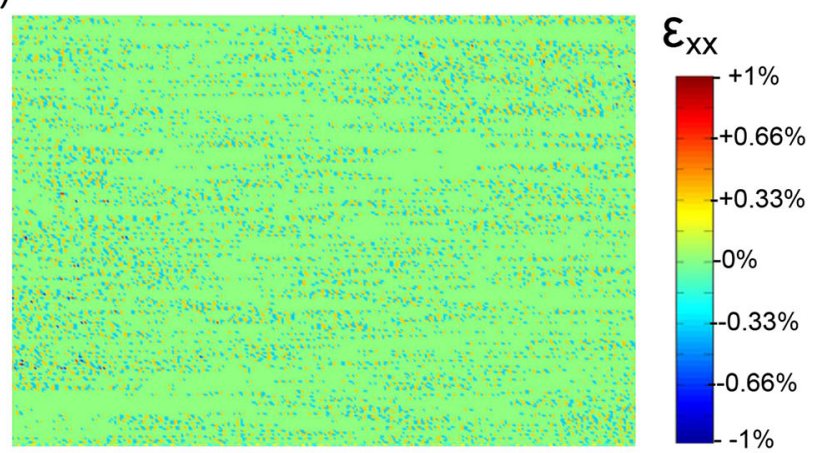

(d)

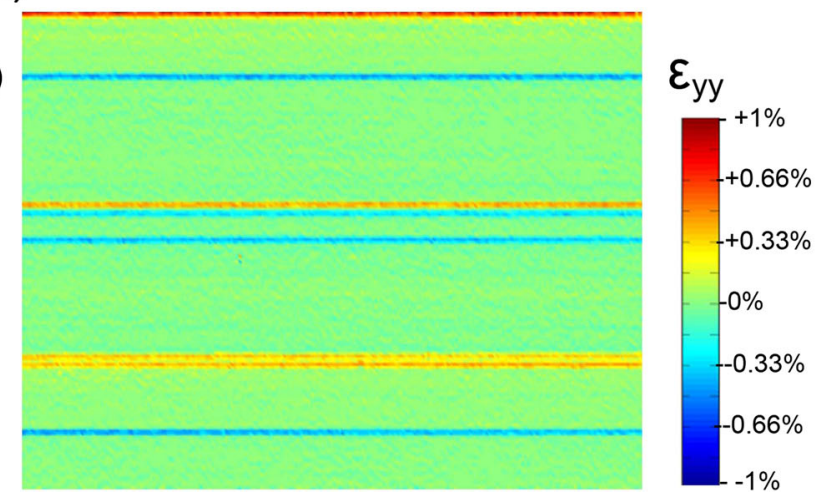

Fig. 8 Drift distortions associated with SEM imaging at magnification of HFW of $85 \mu \mathrm{m}(1500 \times$ mag.) are shown for displacement fields along the horizontal and vertical direction (a) and (c). The displacement steps in the $U_{y}$ field (c) are randomly introduced by the scan generator. The strains maps for $\epsilon_{x x}$ and $\epsilon_{y y}$ associated with the noise induced by drift distortion are calculated in (b) and (d) 
(a)

\section{$0.29 \%$ macrocopic strain Subset : $21 \mathrm{px}(0.4 \mu \mathrm{m})$ Step : 5 px $(104 \mathrm{~nm})$ Strain window : 25 px (0.47 $\mu \mathrm{m})$}

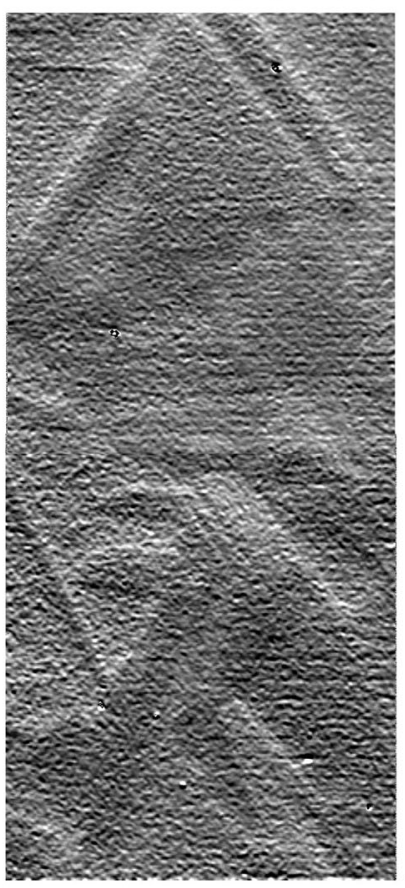

(d) (b) $0.29 \%$ macrocopic strain
Subset : 21 px $(0.4 \mu \mathrm{m})$
Step : 5 px $(104 \mathrm{~nm})$
Filter : 25

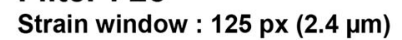

(c)

\section{$0.29 \%$ macrocopic strain Subset : $101 \mathrm{px}(2.09 \mu \mathrm{m})$ Step : 17 px (350 nm) Strain window : $85 \mathrm{px}(1.6 \mu \mathrm{m})$}

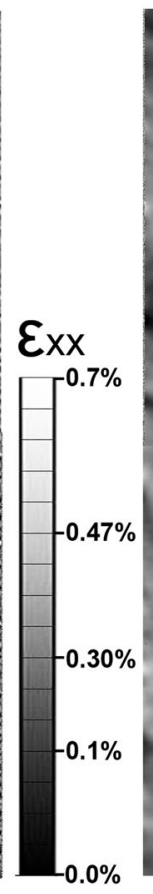

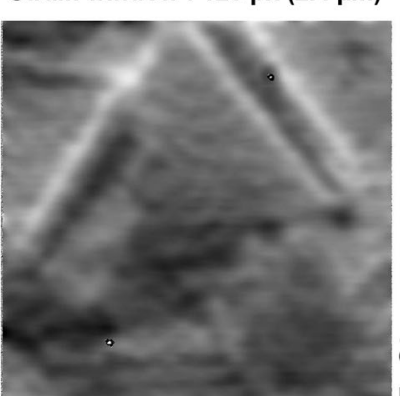

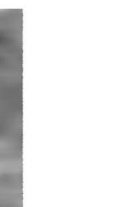

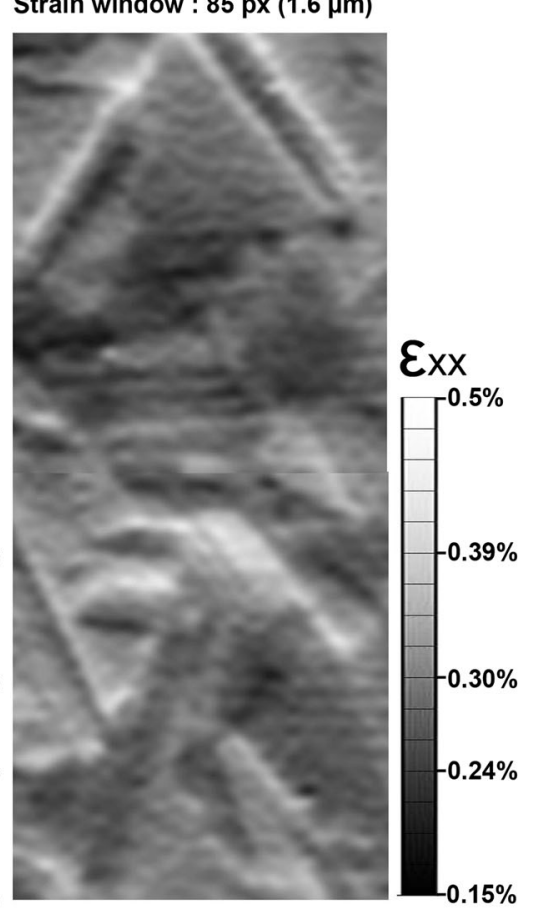

\begin{abstract}
After unloading Subset : $21 \mathrm{px}(0.4 \mu \mathrm{m})$ Step : 5 px $(104 \mathrm{~nm})$ Strain window : $25 \mathrm{px}(0.47 \mu \mathrm{m})$
\end{abstract}

\author{
(e)
}
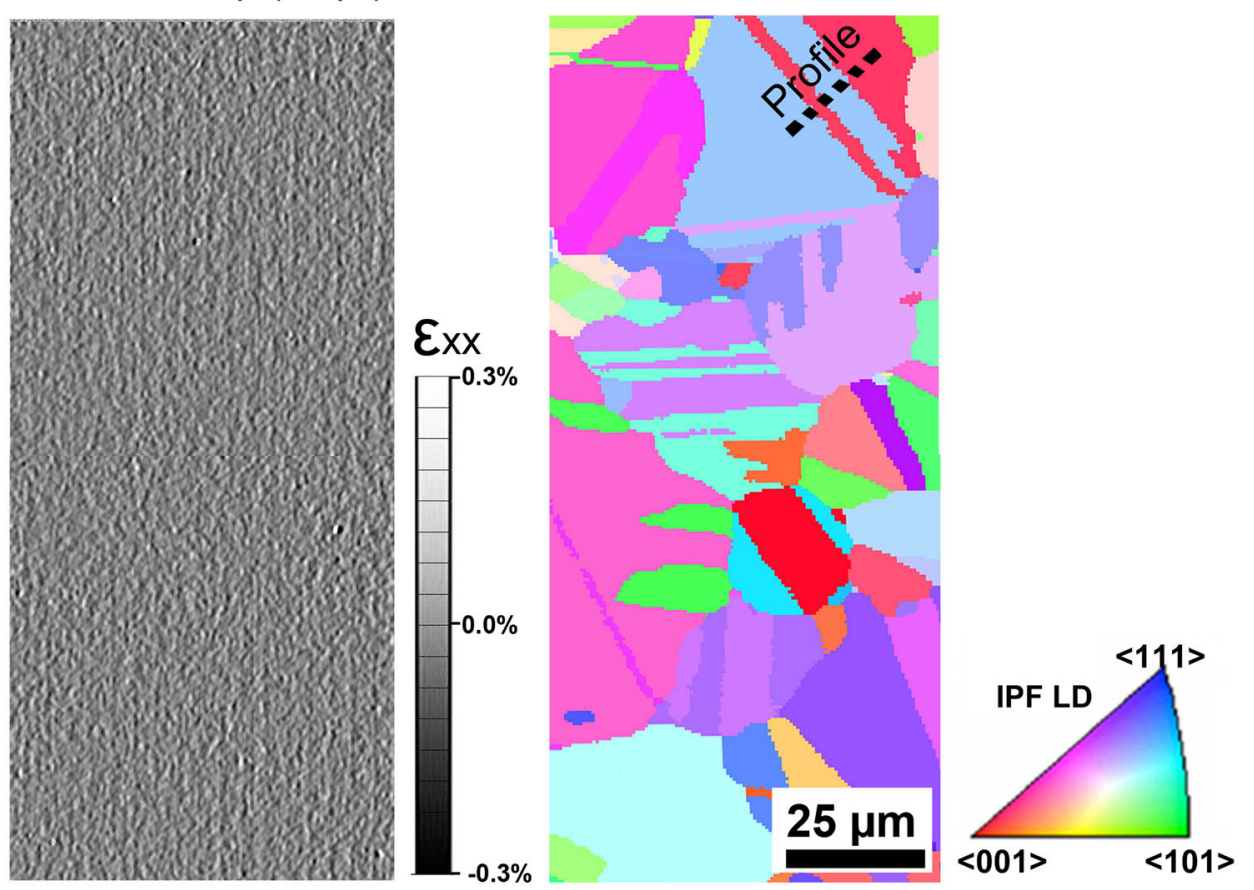

Fig. 9 The strain field $\epsilon_{x x}$ from DIC measurements of two regions imaged at HFW of $85 \mu \mathrm{m}(1500 \times$ mag.) after $0.29 \%$ macroscopic tensile (a-c) strain and after unloading (d). The strain field is resolved for macroscopically elastic loading conditions in (a-c), however filtering of the measurement or applying larger subset and step size improves the signal to noise ratio while limiting some spatial resolution (b,c). An EBSD map is shown in (e) along the loading direction for the same field of view as (a-d) with the location of the profile plotted in Fig. 10 
field being measured. The scan rotation imaging methodology was applied for all images collected at all deformation states during the tensile loading.

\section{Stitching Procedure}

The electron beam and imaging parameters combined with a low HFW enable high strain resolution ( $\epsilon_{x x}$ field) for DIC measurements in the SEM. However, small HFWs necessitate the use of DIC image stitching [35]. Algorithms for the collection of image sets at optimal HFW have been developed for DIC calculations on low distortion images that scale to large sample areas. The iFAST SEM scripting software was used in a FEI Helios microscope to automate the collection of DIC image sets. Images were collected along a sampling grid with $10 \%$ HFW overlap on each bounding border. The microstructure in the center of the field of view of each of the images collected from the unstrained samples was used as a fiducial marker for the alignment of the subsequent strain state image centers using iFAST alignment software. Automated mechanical SEM stage shifts were used to align the strained sample image centers with the unstrained image centers to within $1 \mu \mathrm{m}$. Focus was defined for each image using an autofocusing routine utilizing the pyramid sharpness function [36] for 30 sample images collected at a resolution of 1024 by 884 at a HFW of $25 \mu \mathrm{m}$. Scan distortions induced by the microscope beam scanning raster pattern were reduced by collecting images at both $0^{\circ}$ and $90^{\circ}$ scan rotation and then used independently for the measurement of $\epsilon_{x x}$ and $\epsilon_{y y}$ strains, as described in "SEM Beam Scanning Defects" section. Stitching was performed after DIC strain calculations were made for all image sets. Imaging conditions were optimized for data collection in either the elastic or plastic regime, as discussed in Section "Strain Field in the Nominally Elastic Deformation Regime".

\section{Strain Field in the Nominally Elastic Deformation Regime}

Imaging conditions must be carefully selected for the measurement of strain fields in the nominally elastic deformation regime using the DIC technique, particularly because of the small strains being measured and the distortions present in SEM imaging. Foremost, the speckle pattern size relative with the subset size has a large influence on the ability to collect DIC strain measurements [37], particularly at the subgrain scale for elastic strain field measurements. The DIC subset size and step size must also be optimized to resolve the strain field, as shown in Fig. 9. The strain field obtained with a low subset size $(0.4 \times 0.4 \mu \mathrm{m})$ is presented in Fig. 9(a). The high spatial resolution obtained with these SEM imaging conditions and the subset size comes at the cost of variability in strain measurements, which induce strain errors resulting from SEM distortions and detector noise of about $0.15 \%$ and are of similar magnitude to the strain being measured near $0.30 \%$. When measuring the elastic strain field by DIC, high spatial resolution in the strain maps is not necessary because strain variations are diffuse over the grains when compared to the plastic strain localization along slip. However, the low strains developed by elastic deformation are comparable to the error inherent to DIC with SEM imaging, requiring filtering to increase the signal to noise ratio.

Filters or larger step sizes, both affecting the strain window size, and larger subset sizes that worsen the spatial resolution but resolve the measurements of the strain field can be used for the identification of small strain levels. This is demonstrated by decay filtering of $25 \times 25$ pixels applied in Fig. 9(b) or the use of a larger subset size $(2.09 \times 2.09 \mu \mathrm{m})$ used in Fig. 9(c). A profile of the measured strain across the twin boundary in Fig. 9(e) is shown in Fig. 10 for the DIC parameters used in both Fig. 9(a,b and c), indicating that the strain measurements are equivalent for both the applied spatial filter, Fig. 9(b), and large subset size, Fig. 9(c). However, the peak amplitude of the strain measurements are reduced by increased spatial filtering or a large subset size. The strain map and the strain along the profile depicted in Fig. 9(e) after unloading are shown in Figs. 9(d) and 10 , respectively. The elastic strain localization is no longer observed upon unloading.

\section{$0.29 \%$ macrocopic strain:}

- Subset : $0.4 \mu \mathrm{m}$, step : $104 \mathrm{~nm}$

$\Delta$ Subset : $0.4 \mu \mathrm{m}$, step : $104 \mathrm{~nm}$; filter $25 \mathrm{px}$

After unloading:

Subset : $2.09 \mu \mathrm{m}$, step : $350 \mathrm{~nm}$

Subset : $0.4 \mu \mathrm{m}$, step : $104 \mathrm{~nm}$

- Subset : $0.4 \mu \mathrm{m}$, step : $104 \mathrm{~nm}$; filter $25 \mathrm{px}$

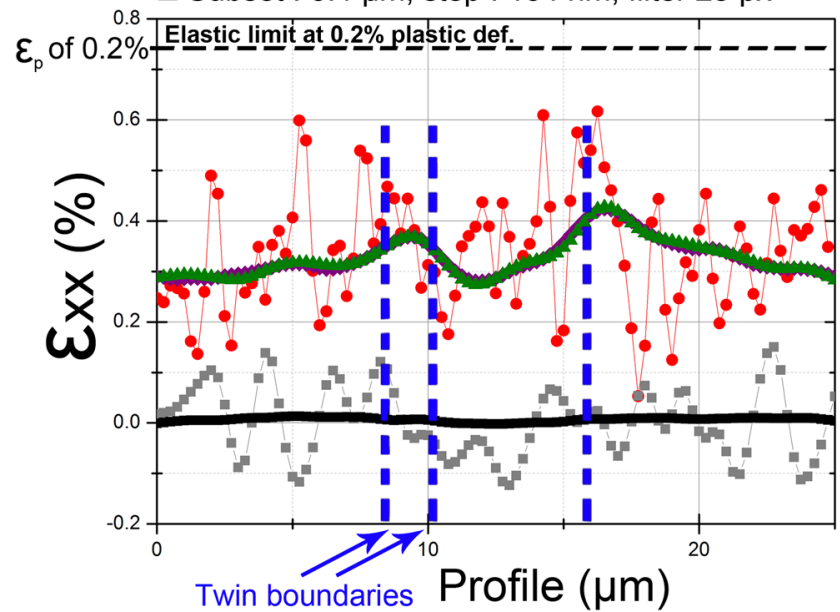

Fig. 10 The strain $\left(\epsilon_{x x}\right)$ profile along the dotted line plotted in Fig. 9(e) for each of the DIC parameters in Fig. 9 after $0.29 \%$ macroscopic tensile strain and after unloading, showing the resolved strain under elastic loading conditions 


\section{Plastic Strain Field}

The calculation of the plastic strain field using DIC measurements requires a different set of parameters when compared to the elastic strain field measurements because the plastic deformation is sharply localized. For the calculation of the elastic strain field, high strain resolution is more important than spatial resolution because of the relatively low amplitude of the strains being measured compared to the SEM distortions and secondary electron detector noise. However, for the calculation of plastic strain fields, spatial resolution becomes important for the details of localization with respect to grain boundary and twin boundary location. For example, a small subset size and step size are required to resolve the location of slip localization in the grain structure shown in Fig. 11. Choosing a large subset size and step size yields a diffuse strain field shown by the white arrow in Fig. 11(b and c), which cannot be related to the boundaries shown in Fig. 11(a). Conversely, the selection of a small subset size and step size accurately places the slip along the expected $\{111\}$ planes in the grains, as shown in Fig. 12, as has also been observed by Di Gioacchino et al. [16]. Moreover, very sharp localization of strain cannot be observed when using large subset and step size as shown by the black arrow in Fig. 11(b and c). The local displacement associated with the strain localization along the slip band at the black arrow, Fig. 11(b), is not captured by the use of large step and subset size in Fig. 11(c).

There exists a critical size of the subset and step above which the plastic strain field is no longer representative. Since plastic deformation mechanisms in this material are at the scale of the slip bands, the strain measurement must be at the same scale. For example, the strain magnitude is demonstrated to be greater by a factor of 2 at the center of the slip band traces when comparing measurements made with optimal and suboptimal DIC parameters, as shown by Fig. 11(b) compared with Fig. 11(c). The selection of suboptimal DIC parameters may lead to incorrect inferences about the spatial localization of strain. The subset size and step sizes used in Fig. 12(a and b) show the sharp localization of the plastic strain along the slip traces, which is not resolved in either larger step sizes shown in Fig. 12(c) or larger subset sizes shown in Fig. 12(d). Quantitatively, the DIC parameters used in Fig. 12(a) resolve a factor of 2 higher strain which is shown to localize on either side of a twin boundary as shown in the Fig. 12(f). The loss

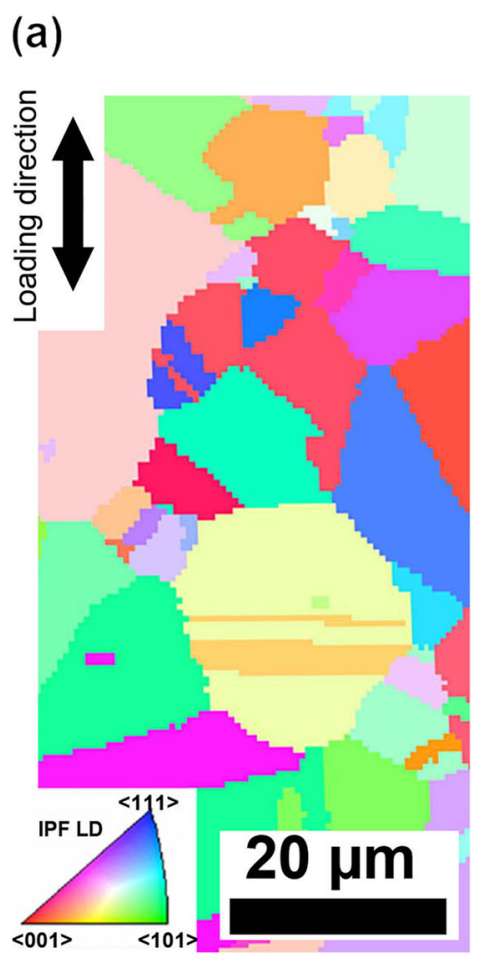

\section{(b)}
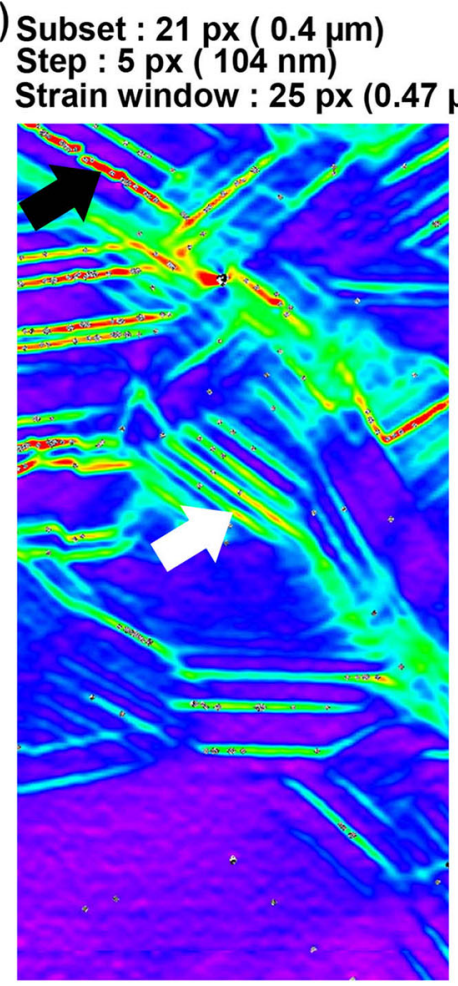

(c) Subset: $111 \mathrm{px}(2.3 \mu \mathrm{m})$
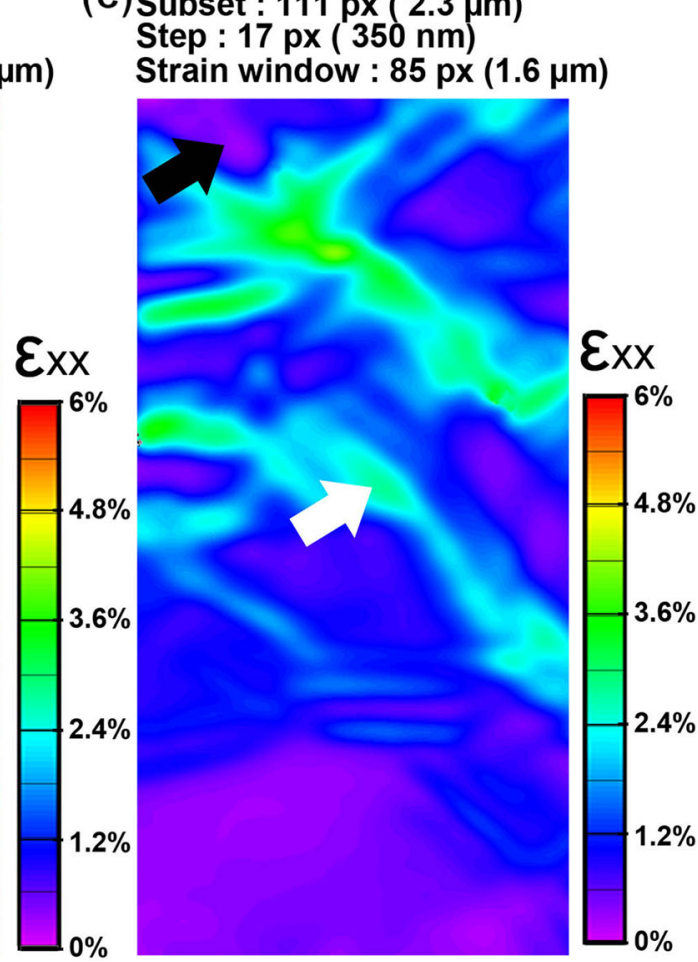

Fig. 11 The strain field $\epsilon_{x x}$ from DIC measurements of a region imaged at a HFW of $85 \mu \mathrm{m}(1500 \times$ mag.) after $0.98 \%$ macroscopic tensile strain. (b) Spatial resolution of the plastic strain localization by slip is permitted by use of a $21 \times 21$ pixel subset size and a $5 \mu \mathrm{m}$ step size. (c) Suboptimal subset and step sizes show a diffuse strain field with reduced spatial localization information. An EBSD IPF map is shown in (a) with reference to the loading direction 
Subset : $21 \mathrm{px}(0.4 \mu \mathrm{m})$
(a) Step : $3 \mathrm{px}(62 \mathrm{~nm})$

Strain window : $15 \mathrm{px}(0.28 \mu \mathrm{m})$

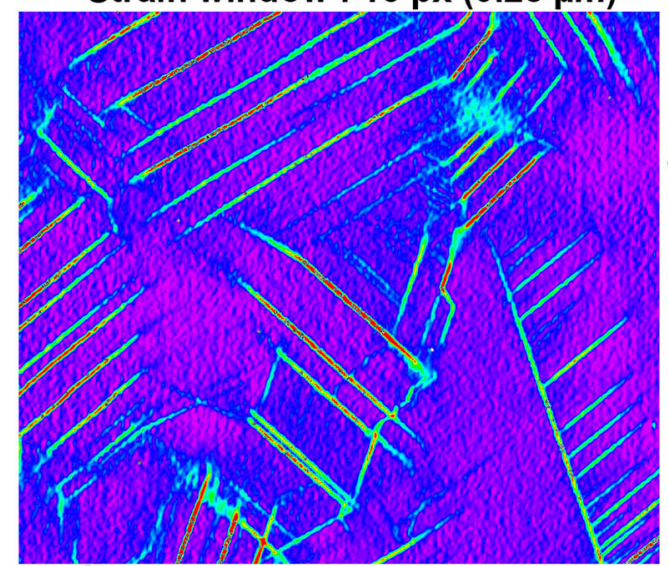

Subset : $21 \mathrm{px}(0.4 \mu \mathrm{m}$
(C) Step : $17 \mathrm{px}(350 \mathrm{~nm})$

Strain window : $85 \mathrm{px}(1.6 \mu \mathrm{m})$

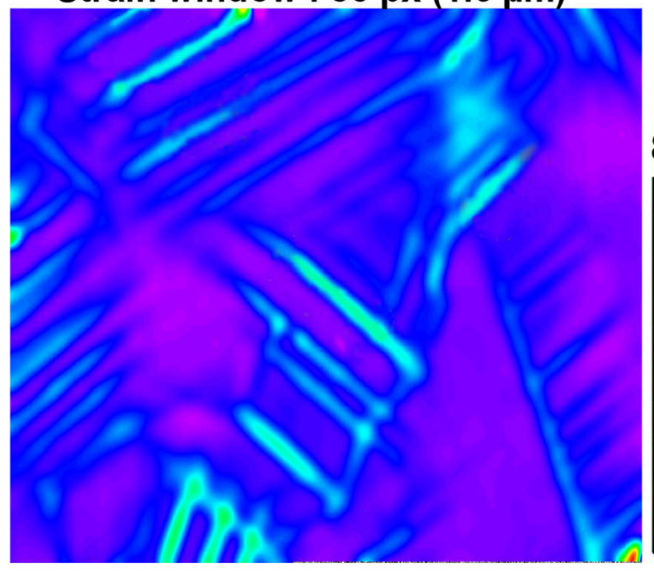

(e)

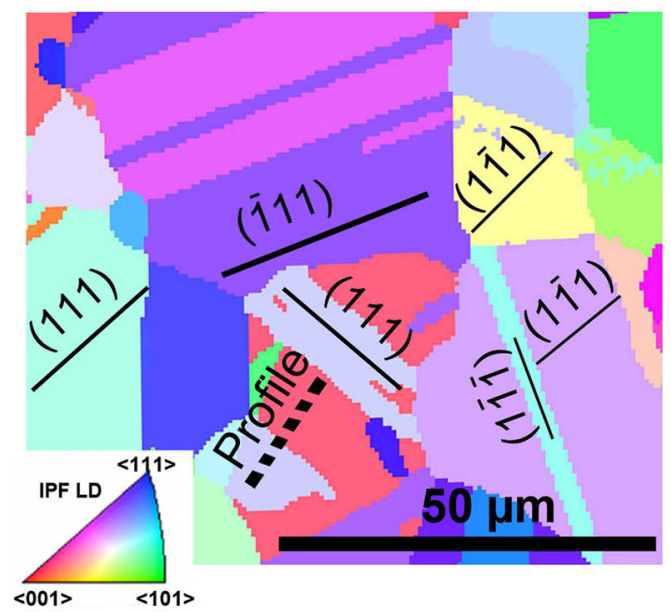

(b) Subset : $21 \mathrm{px}(0.4 \mu \mathrm{m})$

Step : 5 px ( $104 \mathrm{~nm}$ )

Strain window : $25 \mathrm{px}(0.47 \mu \mathrm{m})$

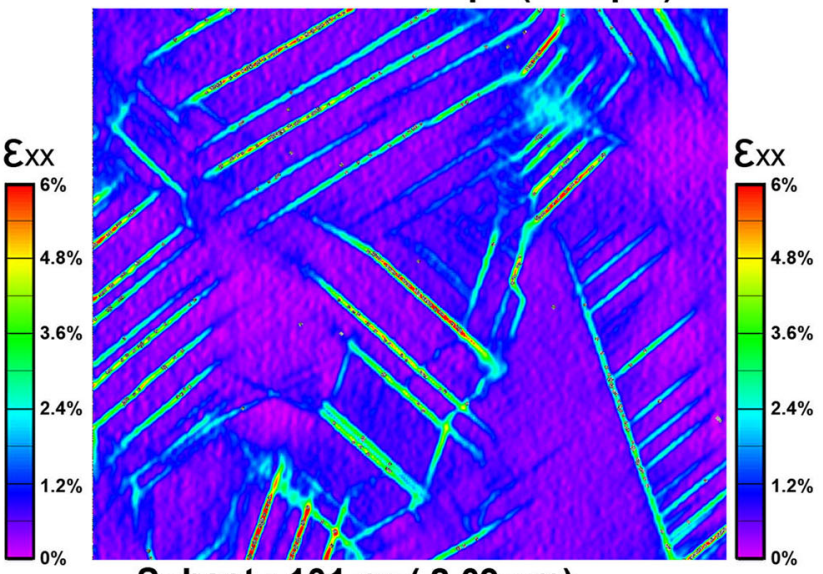

Subset : $101 \mathrm{px}(2.09 \mu \mathrm{m})$

(d) Step : 5 px (104 nm)

Strain window : $25 \mathrm{px}(0.47 \mu \mathrm{m})$

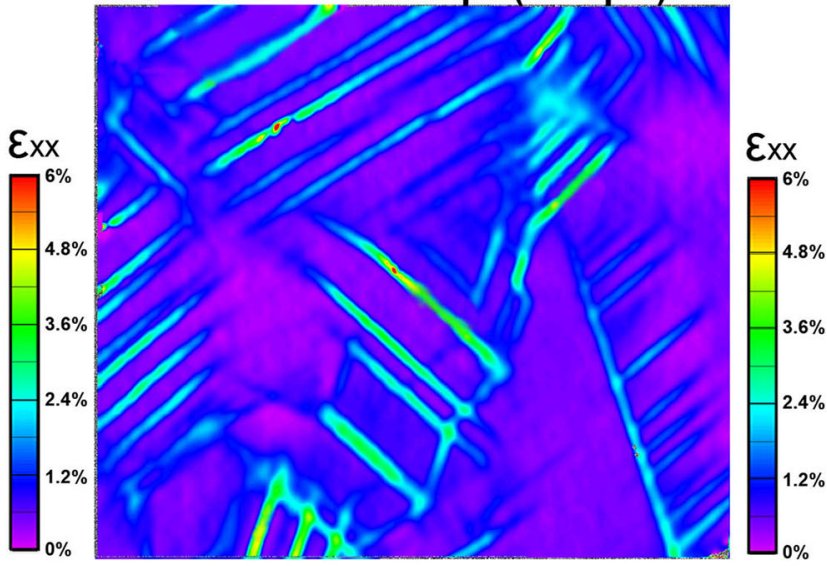

(f)

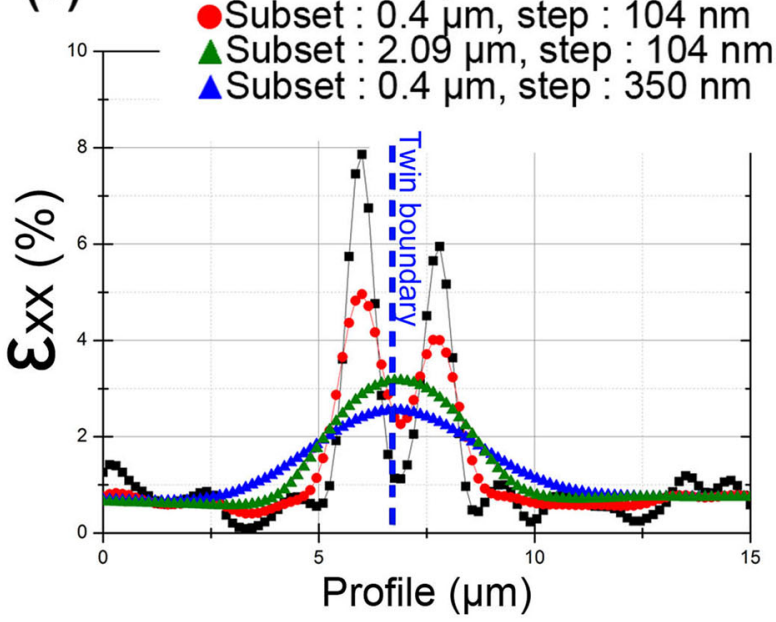

Fig. 12 (a-d) The strain field $\epsilon_{x x}$ from DIC measurements of a region imaged at magnification of HFW $85 \mu \mathrm{m}(1500 \times$ mag.) after $0.98 \%$ macroscopic strain in tension is shown for optimal and suboptimal DIC parameters. (a) The localization of the plastic strain with respect to the microstructure of the sample is permitted by use of a $21 \times 21$ pixel subset size and a 3 pixel step size. (b-d) Increased step sizes show a loss in spatial resolution of the strain localization and a decrease in amplitude of the peak strain. The associated EBSD IPF map referenced along the loading direction is shown in (e). The measured strain $\epsilon_{x x}$ along the profile depicted in (e) for the different DIC parameters is shown in (f). A vertical dotted line indicates the position of the twin boundary with respect to this profile 
(a)

$$
\text { OpenDIC : unfiltered }
$$

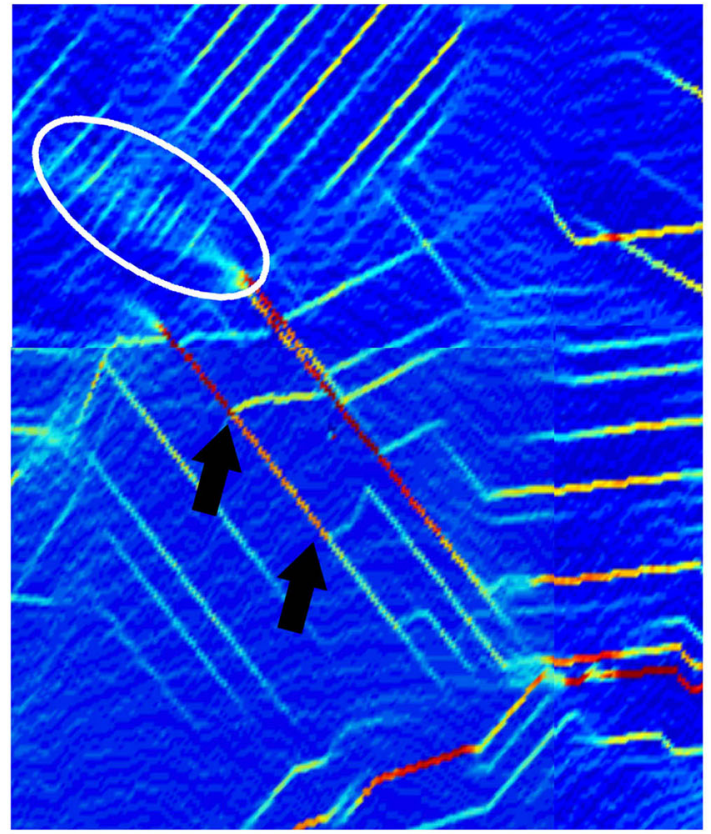

(C)

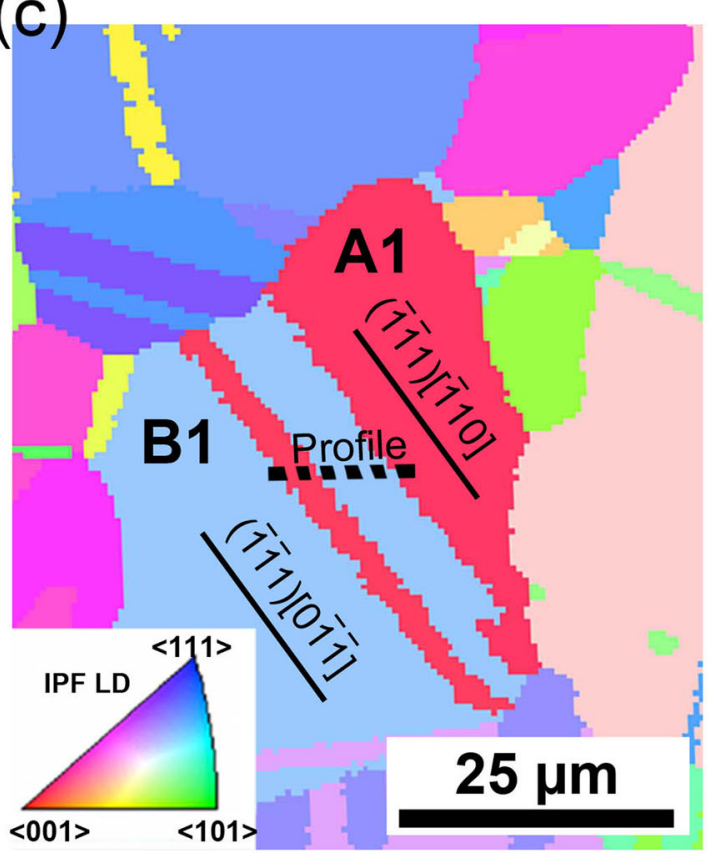

(b)

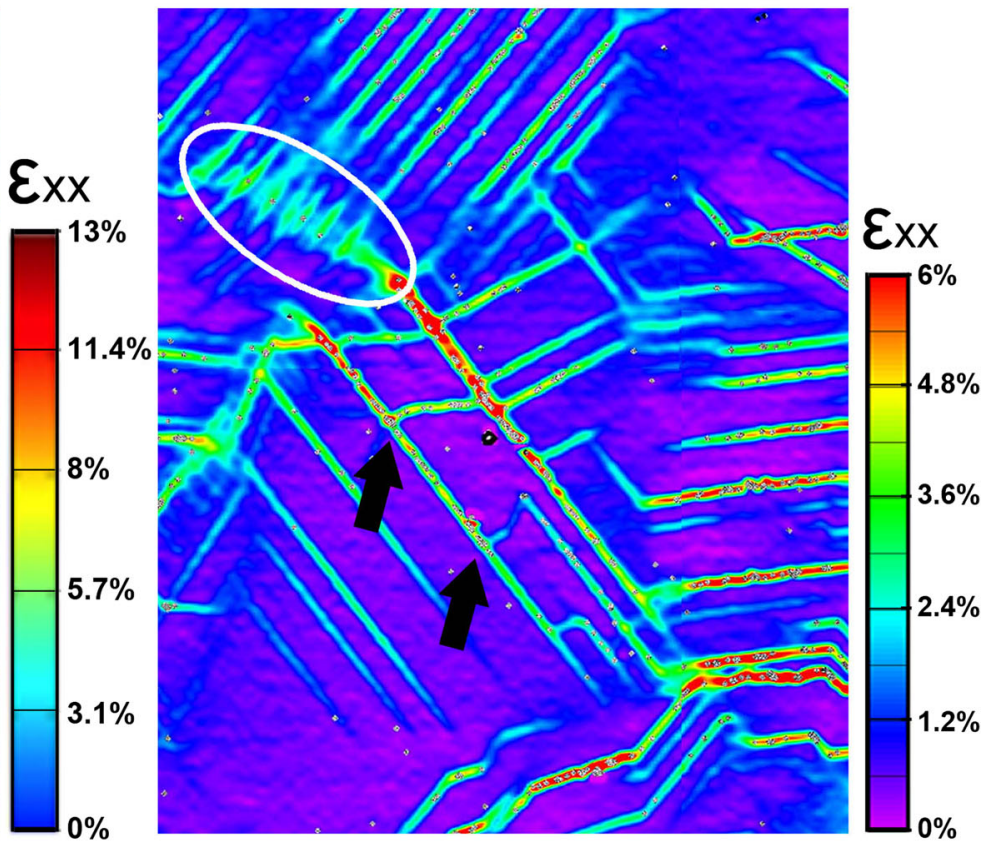

(d)

Unfiltered (OpenDIC)

- Filter : 5 (Vic2D)

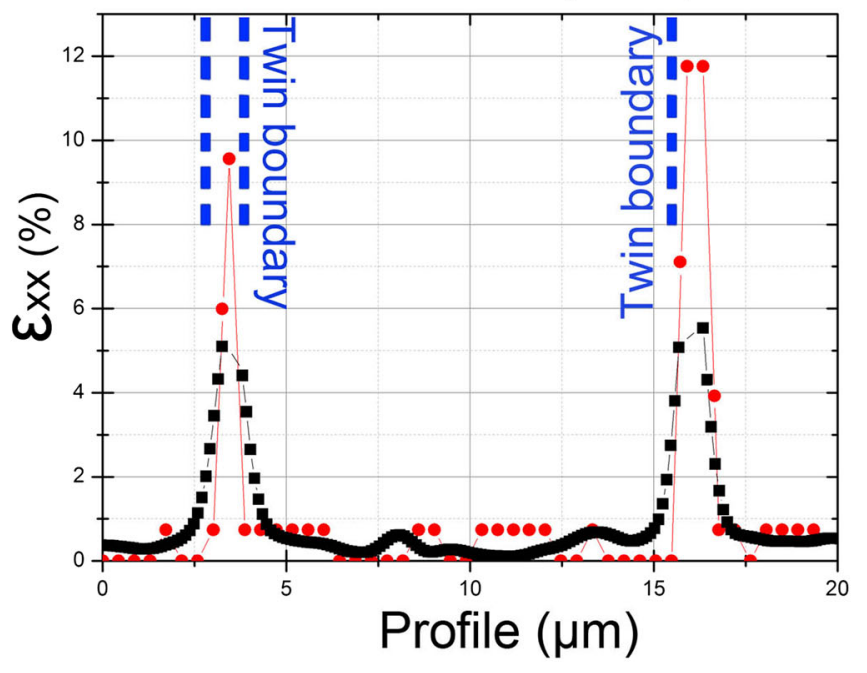

Fig. 13 (a-b) The stitched strain field $\epsilon_{x x}$ from DIC measurements of a region imaged at a HFW of $85 \mu \mathrm{m}(1500 \times$ mag.) after $0.98 \%$ macroscopic tensile strain from DIC with and without the use of a filter, respectively. The associated EBSD IPF map along the loading direction in shown in (c). The vertical direction is the loading direction (x-direction). The strain $\epsilon_{x x}$ along the profile depicted in (c) for the two DIC parameters is shown in (d). The vertical dotted lines indicate the position of the twin boundaries along this profile. Steps in strain localization along the twin boundaries, by slip on a secondary system, are not resolved in (b) compared to (a) as shown near the black arrows. Additionally, the details of plasticity transmitted from the intersection of a highly strained slip band into an adjacent grain, changing the local loading conditions, is resolved in the circled region in white between (b) and (a)

in spatial resolution is also observed when applying filters available in commercial DIC software, e.g. Fig. 13(a and b). For instance, the circled region in Fig. 13(b) shows critical grain configurations leading to the activation of a micro volume [38], which is resolved in Fig. 13(a), where plasticity is transmitted from the intersection of a highly 
strained slip band into the adjacent grain, changing the local loading conditions. The unfiltered strain field data at the black arrows in Fig. 13(a) shows the steps in strain at the intersection of two slip bands, which are reduced in magnitude and spatial resolution compared with the filtered strain data shown in Fig. 13(b). Quantitatively, the refinement of the twin boundary locations with respect to strain localization and the magnitude of the strain localization is plotted in Fig. 13(d) for both the filtered and unfiltered DIC datasets.

Determination of the critical subset and step size for analysis of deformation processes is of primary importance when investigating strain localization during cyclic loading at low stresses. The residual strain field in a René 88DT sample that was fatigue cycled at a macroscopic stress of $758 \mathrm{MPa}$ with fully reversed loading conditions at room temperature using a sub-micron strain window is shown in Fig. 14(b). DIC measurements were performed after unloading the sample. During cycling, strain localization was observed near a twin boundary with a parallel slip configuration, i.e. the twin and associated parent grain exhibit a slip system parallel to the twin boundary. The specifics of strain localization during fatigue in René 88DT are presented in more detail elsewhere [39]. In summary, after a large number of cycles, cracks are detected at the locations of strain localization. The use of suboptimal DIC parameters, such as strain windows or subset sizes larger than microns, do not allow the observation of the residual strain localization after cycling as shown in Fig. 14(c).

\section{Strain Field Measurement Convergence}

The localization of strain depends on the physical processes, such as atomic elastic displacements for elastic strain fields and dislocation motion for plasticity. The speckle pattern and DIC parameters must be adapted to provide resolution at or below the scale of the operation of these physical mechanisms in order to spatially resolve representative strains and strain magnitudes. In the following section we show a methodology to determine the DIC parameters via a convergence metric, in the elastic and plastic regimes for René 88DT alloy.

For an nominally elastically loaded sample at $0.29 \%$ macroscopic tensile strain, DIC measurements for strain window sizes of 0.47-1.88 $\mu \mathrm{m}$ in Fig. 15(a) and (c) show the influence of the DIC parameters on the $\epsilon_{x x}$ strain fields. The profiles shown in Fig. 15(d) were extracted along the line shown in Fig. 15(b) for three strain window sizes. The spatial location and width of the strain localization bands do not change for strain window sizes of 0.47 to $0.94 \mu \mathrm{m}$, and the change in magnitude of the $\epsilon_{x x}$ strain is within the fluctuation in noise in the DIC measurement. The strain localization is located very close to a twin boundary, as shown via EBSD in Fig. 10. At strain window sizes at or larger than $1.88 \mu \mathrm{m}$ the magnitude of the $\epsilon_{x x}$ strain decreases and the profile begins to broaden, indicating a departure from convergence in DIC parameters for elastic strain field measurements as will be detailed more generally presently. (a)

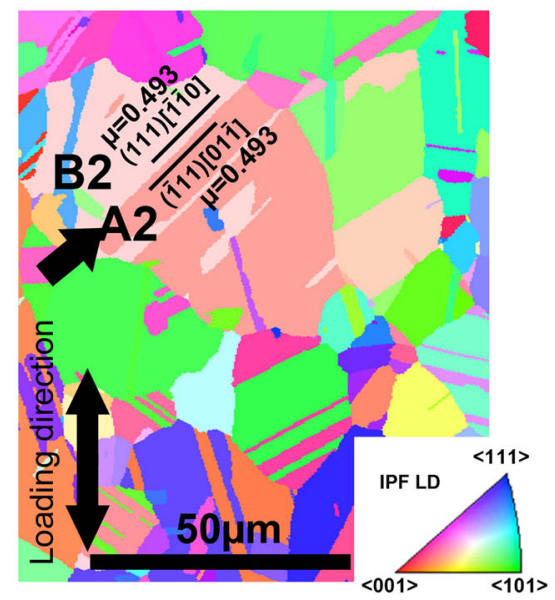

(b) Subset : $21 \mathrm{px}(0.4 \mu \mathrm{m})$ Step : 5 px $(104 \mathrm{~nm})$ Strain window : $25 \mathrm{px}(0.47 \mu \mathrm{m})$

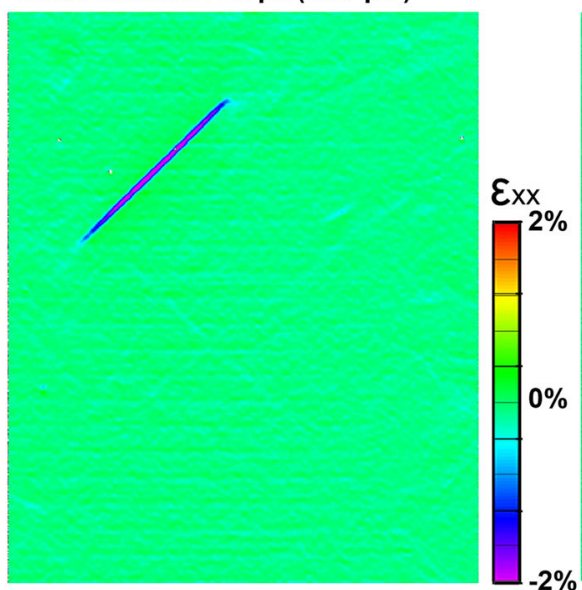

(C) Subset : $111 \mathrm{px}(2.3 \mu \mathrm{m})$

Step : 17 px $(350 \mathrm{~nm})$ Strain window : $85 \mathrm{px}(1.6 \mu \mathrm{m})$

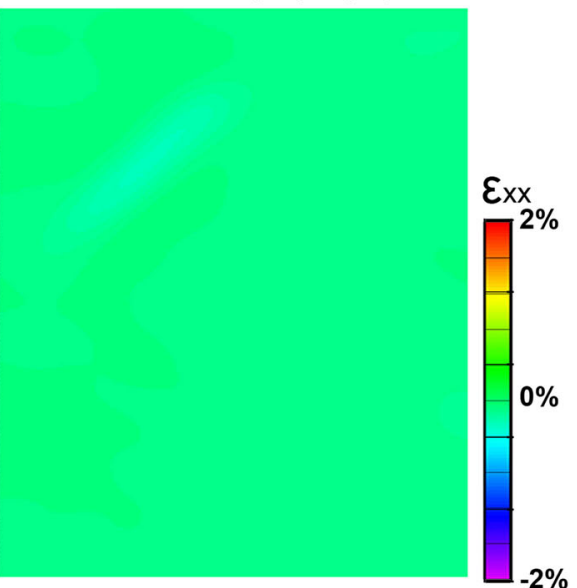

Fig. 14 (b-c) The residual strain field $\epsilon_{x x}$ from DIC measurements of a region imaged at a HFW of $85 \mu \mathrm{m}(1500 \times$ mag.) after fully reversed fatigue cycling at a macroscopic stress of $758 \mathrm{MPa}$ [39]. DIC measurements were made in the unloaded condition. (b) The measurement of the spatial location of plastic strain localization with respect to the sample microstructure (twin boundaries), during fatigue, is permitted by use of a $21 \times 21$ pixel subset size and a 5 pixel step size. (c) DIC strain measurements using suboptimal subset and step sizes result in a diffuse strain field with reduced spatial localization information. The associated EBSD IPF map along the loading direction in shown in (a). The vertical direction is the loading direction (x-direction) 


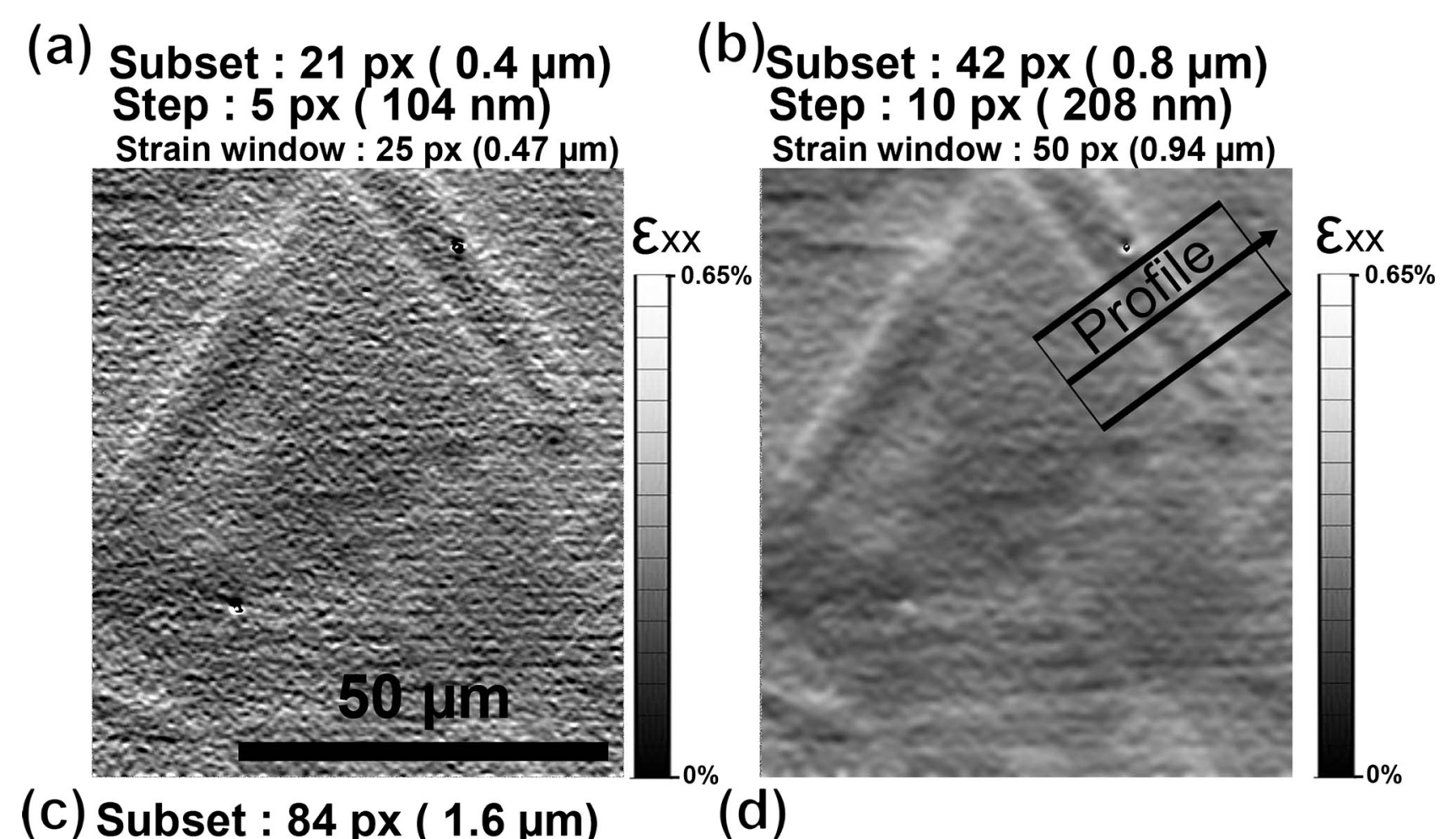

Step : 20 px ( $416 \mathrm{~nm})$

Strain window : $100 \mathrm{px}(1.88 \mu \mathrm{m})$

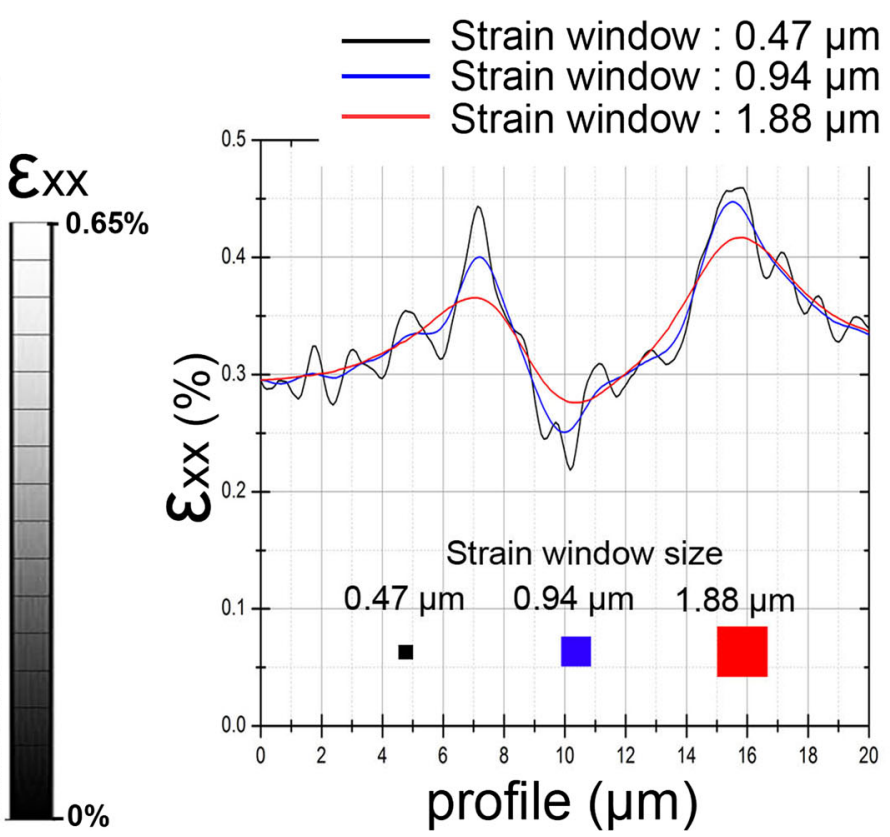

Fig. 15 (a-c) The strain field $\epsilon_{x x}$ from DIC measurements of a region imaged at a HFW of $85 \mu \mathrm{m}(1500 \times$ mag.) after $0.29 \%$ macroscopic tensile strain for strain window sizes ranging from $0.47 \mu \mathrm{m}$ to $1.88 \mu \mathrm{m}$. (d) The $\left(\epsilon_{x x}\right)$ strain profile along the line plotted in $(\mathbf{b})$ and integrated over the box depicted in (b) for strain window sizes of $0.47 \mu \mathrm{m}, 0.94 \mu \mathrm{m}$ and $1.88 \mu \mathrm{m}$

The previous example detailing the resolution of strain localization near a single twin boundary may not be representative of the effect of the DIC parameters on the entire strain measurement. Using Fast Fourier Transforms (FFT), the noise in the strain measurement can be filtered to determine the difference in strain between DIC measurements made while varying DIC parameters. Image difference maps were calculated for strain measurements with strain windows sizes shown in Fig. 16(a and b). The spatial location of strain localization can be observed in Fig. 16(b) before spatial FFT filtering, and more clearly after filtering in the inverse FFT images. Spatial filtering was performed for features smaller than $6.2 \mu \mathrm{m}$ as indicated by circles on the FFT images to decrease the strain noise compared to the strain 
(a)

\section{$0.29 \%$ macroscopic strain}

Diff. strain window 50px (0.94 $\mu \mathrm{m})-25 \mathrm{px}(0.47 \mu \mathrm{m})$
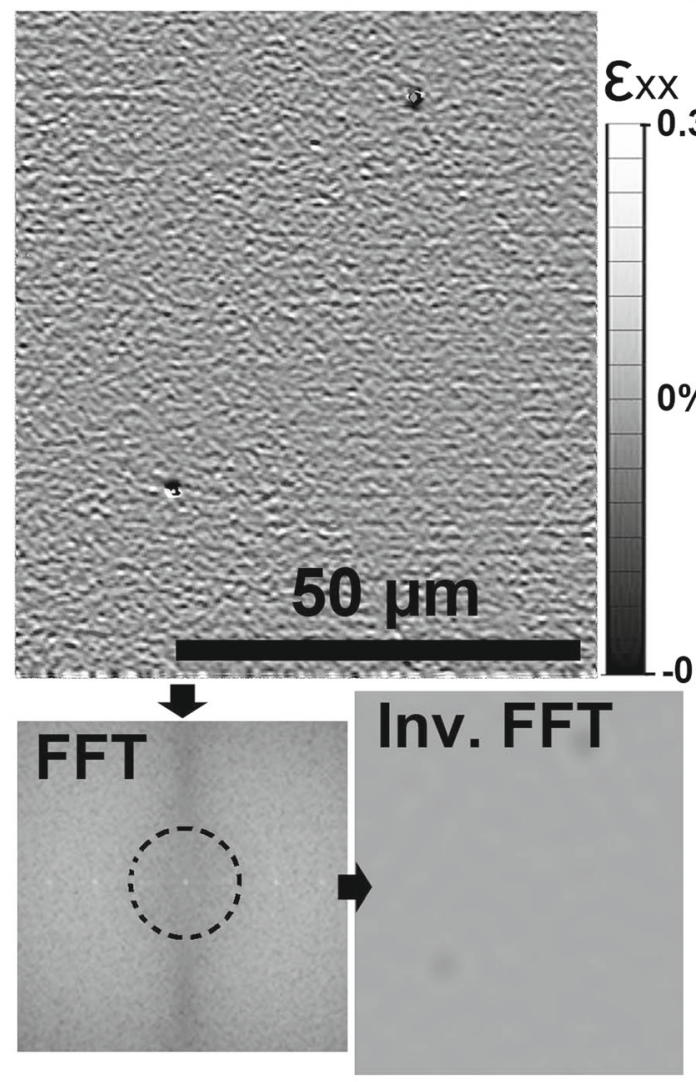

(b)

\section{$0.29 \%$ macroscopic strain}

Diff. strain window 100px (1.88 $\mu \mathrm{m})-50 \mathrm{px}(0.94 \mu \mathrm{m})$
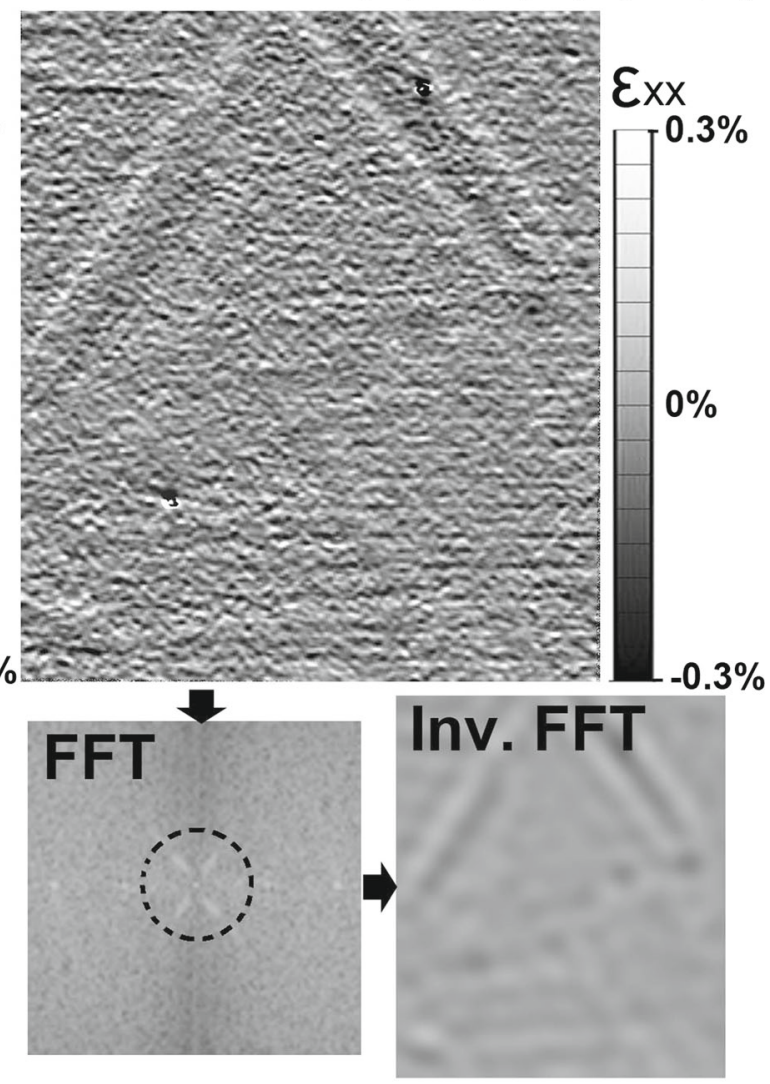

(C)
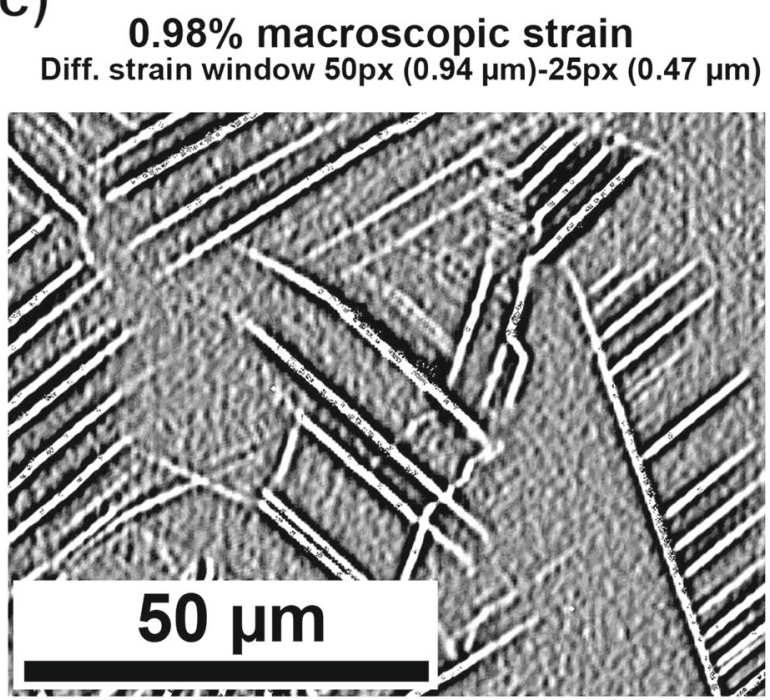

Exx

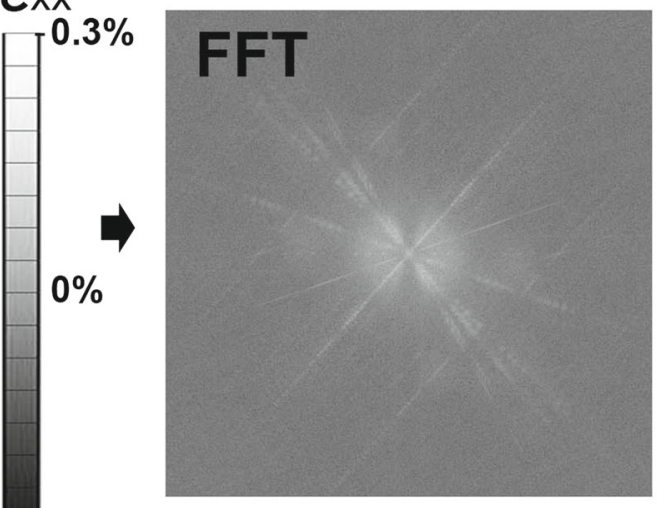

$-0.3 \%$

Fig. 16 The difference between two $\epsilon_{x x}$ strain fields calculated using the DIC parameters shown in the figure captions for a nominally elastically macroscopically loaded sample to $0.29 \%(\mathbf{a}, \mathbf{b})$, and for a sample loaded in the plastic regime to $0.98 \%$ (c) using a HFW of $85 \mu \mathrm{m}(1500 \times$ mag.). The DIC maps between which the differences are calculated are shown in Fig. 15(a-c). Fast Fourier transforms (FFTs) of the difference maps convert the data into reciprocal space where the data is spatially filtered, radially outward from the the overlaid circle, to remove high frequency information. The inverse FFT transforms the data back into real space with the DIC measurement noise removed, now clearly showing the difference in measurements between the two DIC strain maps 
(a)

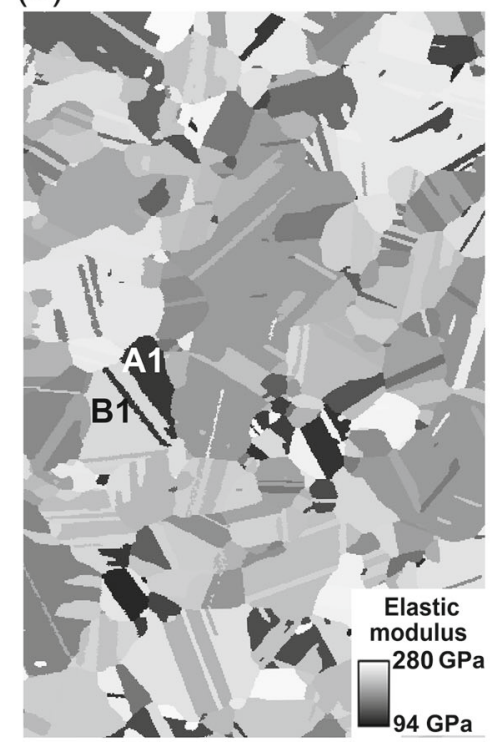

(b)

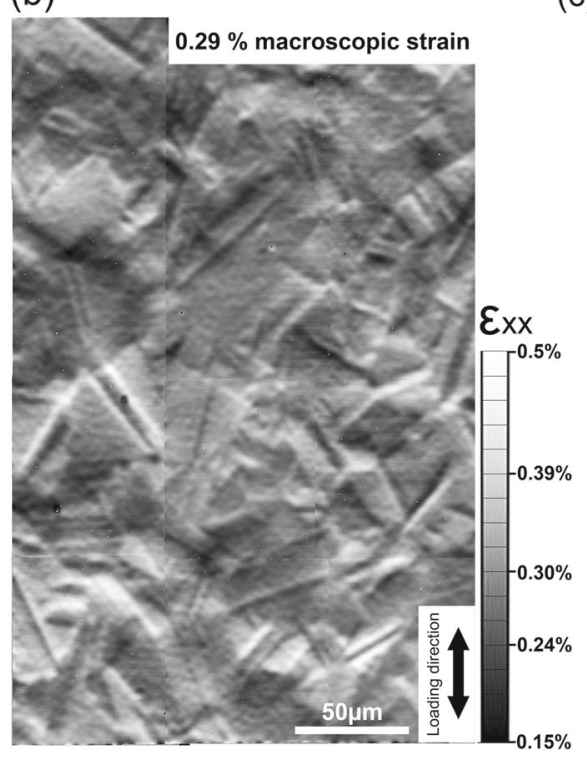

(c) Average strain by grain

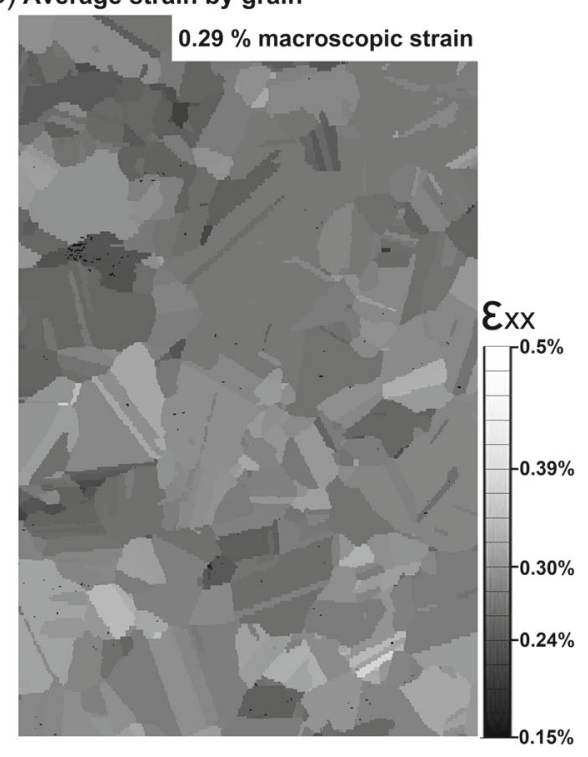

Fig. 17 The stitched strain field $\epsilon_{x x}$ from DIC measurements of regions imaged at $\mathrm{HFW}$ of $85 \mu \mathrm{m}(1500 \times$ mag.) after $0.29 \%$ macroscopic tensile strain is shown in (b). The associated elastic modulus map is shown in (a). The vertical direction is along the loading direction (x-direction). The average strain for each grain extracted from the experimental DIC data and correlated with the EBSD grain boundary information is shown in (c)

measurement. The size of the strain window was increased while monitoring the inverse FFT image of the image difference maps for strains greater than $0.1 \%$, indicating that strain localization broadening was occurring as shown in Fig. 16(b). Within the resolution of the SEM-DIC technique, convergence of the strain window was observed at $0.94 \mu \mathrm{m}$ or below for the macroscopically elastically loaded sample at $0.29 \%$ tensile strain. However, the largest possible strain window size that does not modify the strain profile is selected in order to reduce the noise in the DIC measurement.

An analysis of the DIC parameters used for strain measurement in the plastic regime at $0.98 \%$ was also performed. The image difference map between 0.94 and $0.47 \mu \mathrm{m}$ in Fig. 16(c) shows changes in the spatial width of strain localization and in the peak strain values, indicating that the DIC parameters have not converged for the plastic localization mechanisms present in this material. Nevertheless, the location of the highly localized slip event can be located spatially with resolution $<0.47 \mu \mathrm{m}$ and improvement in the estimate of maximum strain by a factor of 2 to 4 when comparing measurements made using strain window sizes of 0.47 with $1.60 \mu \mathrm{m}$. Therefore, the present SEM DIC resolution provides an accurate measurement of the spatial location of the plastic strain, but does not capture the strain localization magnitude because the localization occurs at the scale of dislocation motion in slip bands observed via TEM in previous work $[24,40]$.

\section{Results and Discussion}

The SEM DIC technique enabled the measurement of strain heterogeneities introduced by elastic and plastic deformation at the $\mu \mathrm{m}$-scale in a polycrystalline material. The results demonstrate strain localization in a René 88DT nickel-base superalloy during the nominally elastic deformation regime (0.29\% macroscopic strain) and at higher levels of plastic deformation (0.98\% macroscopic strain). SEM DIC was independently performed on 12 regions of interest that were then stitched together to obtain the strain maps in the nominally elastic (Fig. 17b) and plastic (Fig. $18 \mathrm{~b}$ and c) deformation regimes.

\section{Elastic Deformation}

The strain field measurements made using DIC for René 88DT samples that were nominally loaded elastically to $0.29 \%$ macroscopic strain show enhanced localization near twin boundaries in grains with high elastic modulus variation along the loading direction. For example, Fig. 17(b) shows the axial strain $\epsilon_{x x}$ after $0.29 \%$ macroscopic strain with enhanced strain between grains A1 and B1 shown in Fig. 17(a), which have high and low elastic moduli along the loading direction. The elastic modulus map is given in Fig. 17(a), which was calculated from the average crystallographic orientation of each grain along the loading direction using the elastic constants for a Ni-base alloy. Specifically, Ni-base alloys have a high Zener elastic anisotropy ratio 


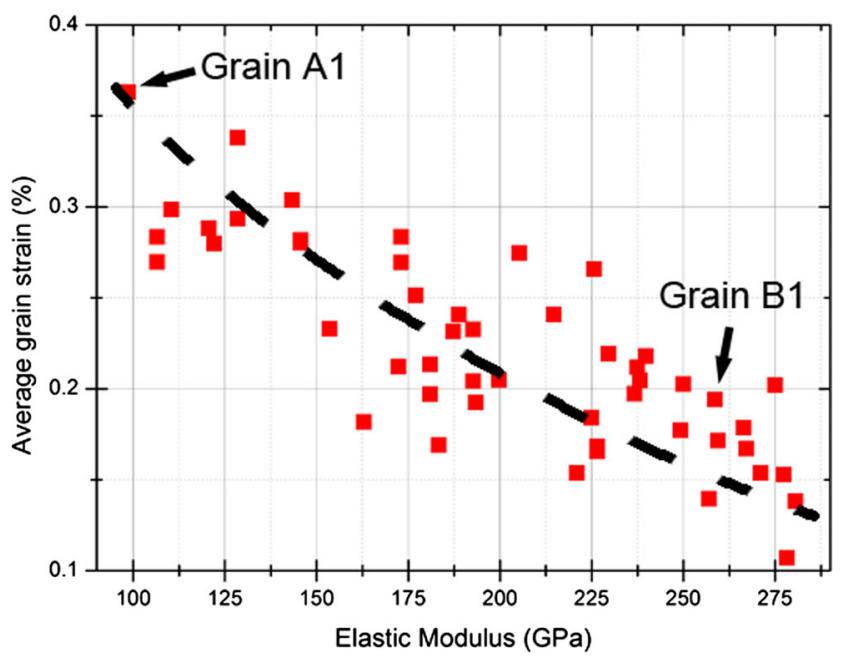

Fig. 18 The average $\epsilon_{x x}$ strain obtained by DIC measurements for each grain after $0.29 \%$ macroscopic strain in tension according to their elastic modulus along the loading direction is shown. The 50 largest grains from the map in Fig. 17 are included in this plot

near 2.5 , owing to the high elastic modulus variability as a function of grain orientation. Grains with a low elastic modulus (such as the grain labeled A1 in Fig. 17(b)) exhibited high local strains, while grains with high elastic modulus showed low local strains. The grains with low elastic modulus along with a large difference in elastic moduli across a twin boundary showed particularly enhanced strain localization (e.g. grains in Fig. 17(a) with large internal greyscale difference). This strain map was obtained from SEM imaging at a HFW of $85 \mu \mathrm{m}$ and using DIC parameters of a $21 \times 21$ pixel subset $(0.4 \times 0.4 \mu \mathrm{m})$ and a 5 pixel
(104 $\mu \mathrm{m})$ step size. In addition, a $25 \times 25$ subset decay kernel matrix was used to resolve strain values. As such, the magnitude of the strains may be slightly underestimated, however the localizations are qualitatively accurate. The average $\epsilon_{x x}$ strain was calculated from the DIC strain measurements for each grain as determined by EBSD and is shown in Fig. 17(c). An inverse correlation can be observed between the average strain per grain and the elastic modulus per grain.

Elevated local strains were observed in grains with low elastic modulus. The dependence of local strain on the local elastic modulus is reported in Fig. 18, where the average strain for each grain is plotted against the elastic modulus of the grain relative to the loading direction. The local strain values within a $2 \mu \mathrm{m}$ wide band around the grain edges were excluded from this analysis to minimize the effect of heterogeneities induced by grain boundaries. Significant heterogeneities are visible in Fig. 13(b) at the grain boundaries when the difference in the elastic modulus across the boundary is large. The strain increased locally in the vicinity of the twin boundaries between the grains A1 and B1 in Fig. 17(a). This local increase in strain is associated with the elastic anisotropy assotiated with the twin boundary [41].

\section{Plastic Deformation}

The strain resolution enabled by the SEM DIC technique offers the ability to capture the details of plastic deformation process at the $\mu \mathrm{m}$-scale in a polycrystalline material. (a)

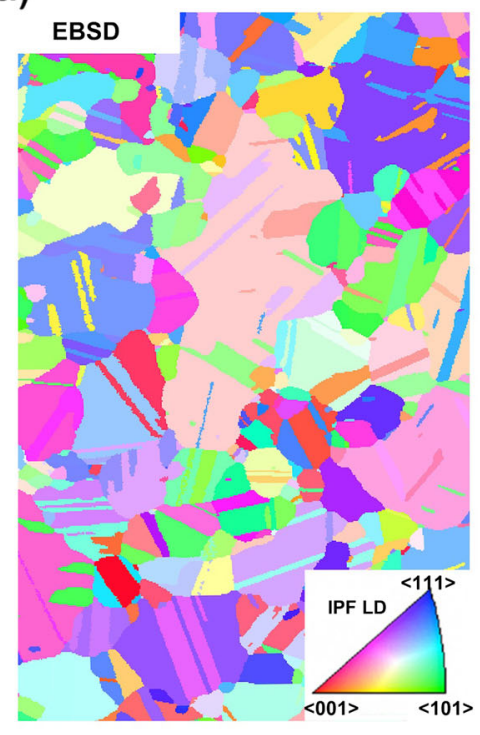

(b)

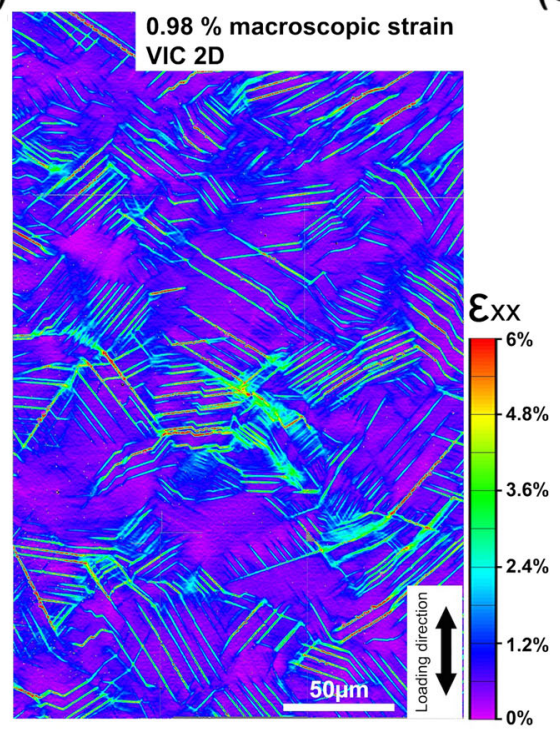

(c)

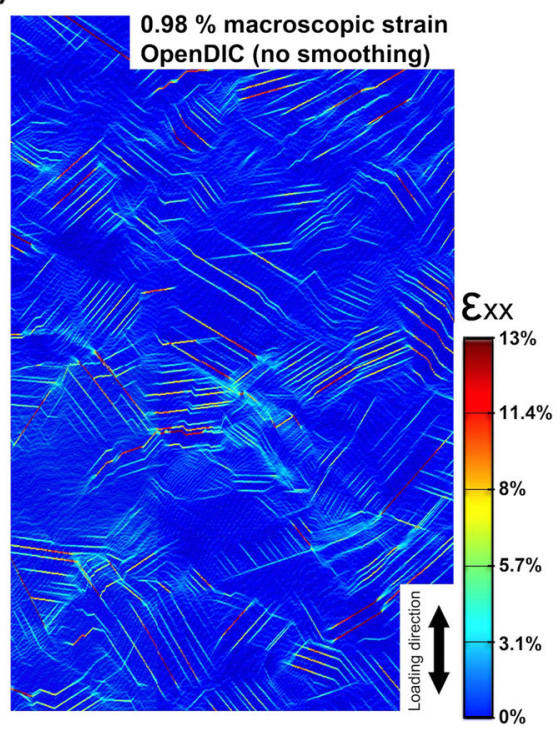

Fig. 19 (b-c) The stitched strain field $\epsilon_{x x}$ from DIC measurements of regions imaged at HFW of $85 \mu \mathrm{m}(1500 \times$ mag.) after $0.98 \%$ macroscopic tensile strain from DIC with and without the use of image filtering, respectively. The associated EBSD IPF map referenced along the loading direction (x-direction) is shown in (a). The vertical direction is parallel with the loading direction 
DIC measurements in Fig. 19(b) and (c) show the $\epsilon_{x x}$ strain field after $0.98 \%$ macroscopic strain using both Vic 2D 2009 and OpenDic, respectively. An EBSD map of the DIC measurement area is given in Fig. 19(a), which shows the locations of the twin and grain boundaries. The strain map was obtained from SEM imaging at HFW of $85 \mu \mathrm{m}$ with DIC parameters of $21 \times 21$ pixels subset $(0.4 \times 0.4 \mu \mathrm{m})$ and a 5 pixel (104 nm) step. Vic 2D measurements additionally implemented a $5 \times 5$ subset decay kernel matrix (minimum filtering) [30]. The strain map in the circled region in Fig. 13( $a$ and $b$ ) shows grain configurations that lead to the activation of levels of high localized plasticity induced by constrained slip in the neighboring grain [38]. Furthermore, the black arrows in Fig. 13(a and b) indicate the locations of secondary slip, which lead to strain relaxation on the primary slip system.

A detailed study of plastic deformation in René 88DT using these sub-grain DIC measurements is reported elsewhere [40]. In brief, bands of concentrated strain are observed during plastic deformation along $\{111\}$ slip

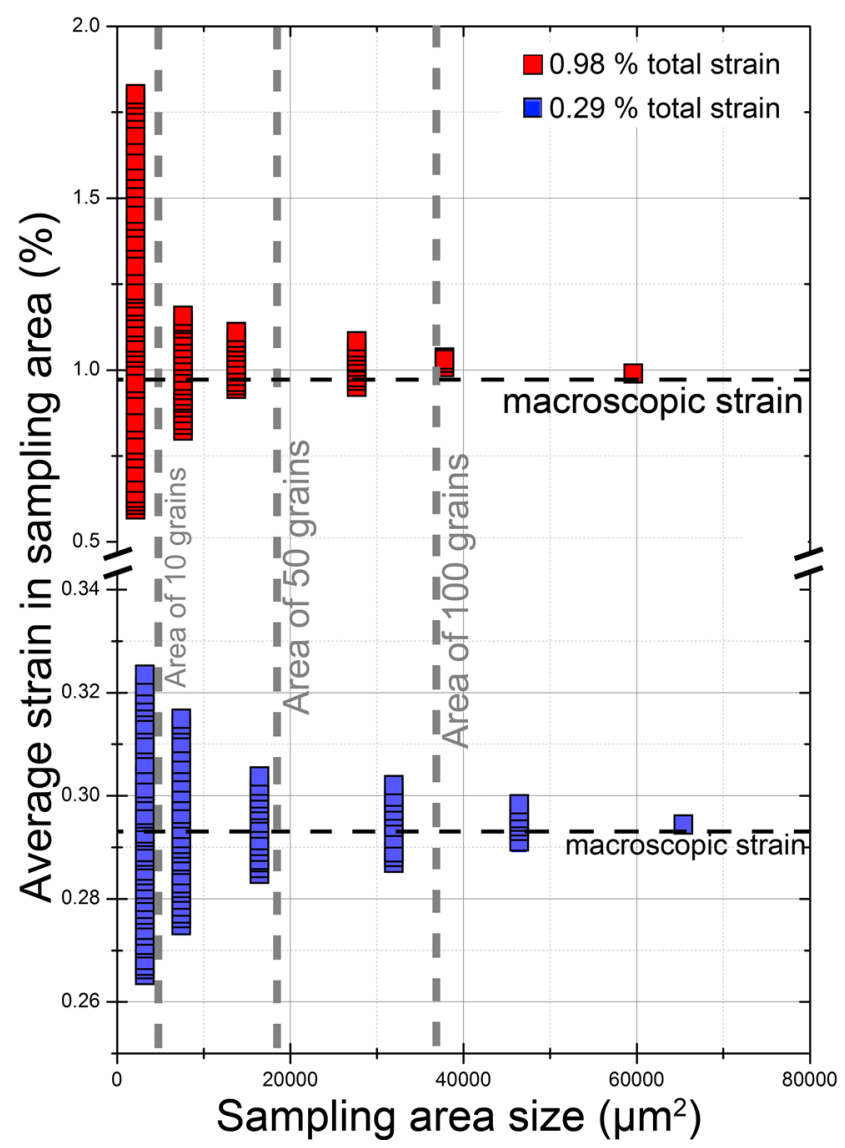

Fig. 20 (a) The average, maximum and minimum $\epsilon_{x x}$ strain obtained by DIC measurements for each region of interest imaged at HFW of 85 $\mu \mathrm{m}(1500 \times$ mag.) after $0.29 \%$ and $0.98 \%$ macroscopic tensile strain. (b) The average $\epsilon_{x x}$ strain according the surface area analyzed by DIC measurement after $0.29 \%$ and $0.98 \%$ macroscopic tensile strain systems, as shown in Figs. 12 and 13. For $98 \%$ of the investigated grains, bands of concentrated strain corresponded to the surface traces of the slip system with the highest Schmid factor based on the loading direction. A single band of concentrated strain was observed in most grains, with few grains showing evidence of secondary bands. Of particular interest, significant strain localization appeared in bands near, but not at, twin boundaries (see grain A1 and B1 in Fig. 13) as observed in TEM micrographs showing a shear offset $[2,40]$.

\section{Strain Distribution and the Role of the Polycrystalline Microstructure}

In order to evaluate the dependence of the strain localization on local microstructure, randomly sampled regions were extracted from the full DIC datasets and variability in the average strain per sampling area size was measured. Fig. 20(b) shows the average strain $\epsilon_{x x}$ along the loading direction plotted as a function of the size of randomly selected regions that were sampled from the full DIC strain map. For comparison, the regions with sizes that correspond to measurements from 10, 50 and 100 grains are given in Fig. 20(b) with vertical dashed lines. At the full DIC dataset size, the average strain is within $2 \%$ of the macroscopic strain measurement. The macroscopic strain can be accurately resolved at small sampled region sizes where the average strain converges. For example, at a sampling size of 50 grains or more the average strain converges to within $10 \%$ of the macroscopic strain. The ability to gather such information on variability of strain distributions as a functions of microstructural volumes is a critical input for mechanical property models for this superalloy material. Such information is also expected to be useful in predicting the property "minimums" and variability for a broader spectrum of polycrystalline materials [42].

\section{Conclusions}

The strain resolution enabled by the combination of SEM and DIC offers the ability to capture strain heterogeneities introduced by deformation at the $\mu \mathrm{m}$-scale. Use of the subgrain microstructure as a speckle pattern permits the resolution of tensile strains larger than $0.15 \%$. The DIC parameters must be carefully chosen (subset size, step size and filtering) to match the type of strain field being measured (nominally elastic or plastic deformation regime) in polycrystalline materials. Special attention must be paid in the DIC measurements of the plastic deformation field, where the spatial resolution of strain localization must be at the same scale of deformation mechanisms such as slip bands. Critical subset and step sizes are presented, beyond 
which the plastic strain field is not representative and can induce incorrect inferences about the spatial localization of strain. In René 88DT superalloy, significant strain localization is observed near twin boundaries during elastic and plastic deformation.

Acknowledgments The authors gratefully acknowledge the support of GE Global Research and appreciate useful discussions with J. Laflen, A. Loghin, S. Daly, and W. LePage. Remco Guerts (FEI) is also acknowledged for his iFAST contributions and support. The Air Force Center of Excellence (Grant \# FA9550-12-1-0445) is also acknowledged for their support. Nicolas Vanderesse is also acknowledged for the development of the OpenDIC software.

\section{References}

1. Miao J, Pollock TM, Jones JW (2009) Crystallographic fatigue crack initiation in nickel-based superalloy René 88DT at elevated temperature. Acta Mater 57(20):5964-5974. doi:10.1016/j.actamat.2009.08.022. http://www.sciencedirect. com/science/article/pii/S1359645409005357

2. Miao J, Pollock TM, Jones JW (2012) Microstructural extremes and the transition from fatigue crack initiation to small crack growth in a polycrystalline nickel-base superalloy. Acta Mater 60(6-7):2840-2854. doi:10.1016/j.actamat.2012.01.049. http:// www.sciencedirect.com/science/article/pii/S1359645412000870

3. Cerrone A, Spear A, Tucker J, Stein C, Rollett A, Ingraffea A (2013). In: Materials Science and Technology (MS\&T) Conference

4. Stein CA, Cerrone A, Ozturk T, Lee S, Kenesei P, Tucker H, Pokharel R, Lind J, Hefferan C, Suter RM, Ingraffea AR, Rollett AD (2014) Fatigue crack initiation, slip localization and twin boundaries in a nickel-based superalloy. Curr Opinion Solid State Mater Sci 18(4):244-252. doi:10.1016/j.cossms.2014.06.001. http://www.sciencedirect.com /science/article/pii/S1359028614000370. Slip Localization and Transfer in Deformation and Fatigue of Polycrystals

5. Zhang M, Bridier F, Villechaise P, Mendez J, McDowell D (2010) Simulation of slip band evolution in duplex Ti-6Al4V. Acta Mater 58(3):1087-1096. doi: 10.1016/j.actamat. 2009.10.025. http://www.sciencedirect.com/science/article/pii/ S1359645409007198

6. Zhao Z, Ramesh M, Raabe D, Cuitiño A, Radovitzky R (2008) Investigation of three-dimensional aspects of grain-scale plastic surface deformation of an aluminum oligocrystal. Int J Plast 24(12):2278-2297. doi:10.1016/j.ijplas.2008.01.002. http://www. sciencedirect.com/science/article/pii/S074964190800020X

7. Merzouki T, Collard C, Bourgeois N, Zineb TB, Meraghni F (2010) Coupling between measured kinematic fields and multicrystal \{SMA\} finite element calculations. Mech Mater 42(1):72-95. doi:10.1016/j.mechmat.2009.09.003. http://www. sciencedirect.com/science/article/pii/S0167663609001562

8. Héripré E, Dexet M, Crépin J, Gélébart L, Roos A, Bornert M, Caldemaison D (2007) Coupling between experimental measurements and polycrystal finite element calculations for micromechanical study of metallic materials. Int J Plast 23(9):15121539. doi:10.1016/j.ijplas.2007.01.009. http://www.sciencedirect. com/science/article/pii/S074964190700006X

9. Walley J, Wheeler R, Uchic M, Mills M (2012) In-situ mechanical testing for characterizing strain localization during deformation at elevated temperatures. Exp Mech 52(4):405-416. doi:10.1007/s11340-011-9499-7
10. Carroll JD, Abuzaid W, Lambros J, Sehitoglu H (2013) High resolution digital image correlation measurements of strain accumulation in fatigue crack growth. Int J Fatigue 57:140-150. doi:10.1016/j.ijfatigue.2012.06.010. http://www.sciencedirect. com/science/article/pii/S0142112312002113. Fatigue and Microstructure: A special issue on recent advances

11. Carroll J, Clark B, Buchheit T, Boyce B, Weinberger C (2013) An experimental statistical analysis of stress projection factors in $\{\mathrm{BCC}\}$ tantalum. Mater Sci Eng A 581:108118. doi:10.1016/j.msea.2013.05.085. http://www.sciencedirect. com/science/article/pii/S0921509313006515

12. Raabe D, Sachtleber M, Zhao Z, Roters F, Zaefferer S (2001) Micromechanical and macromechanical effects in grain scale polycrystal plasticity experimentation and simulation. Acta Mater 49(17):3433-3441. doi:10.1016/S1359-6454(01)00242-7. http:// www.sciencedirect.com/science/article/pii/S1359645401002427

13. Delaire F, Raphanel J, Rey C (2000) Plastic heterogeneities of a copper multicrystal deformed in uniaxial tension: experimental study and finite element simulations. Acta Mater 48(5):1075-1087. doi:10.1016/S1359-6454(99)00408-5. http:// www.sciencedirect.com/science/article/pii/S1359645499004085

14. Jin H, Lu WY, Haldar S, Bruck H (2011) Microscale characterization of granular deformation near a crack tip. J Mater Sci 46(20):6596-6602. doi:10.1007/s10853-011-5608-3

15. Kammers A, Daly S (2013) Digital image correlation under scanning electron microscopy: methodology and validation. Exp Mech 53(9):1743-1761. doi:10.1007/s11340-013-9782-x

16. Di Gioacchino F, Quinta da Fonseca J (2013) Plastic strain mapping with sub-micron resolution using digital image correlation. Exp Mech 53(5):743-754. doi:10.1007/s11340-012-9685-2

17. Patriarca L, Abuzaid W, Sehitoglu H, Maier HJ (2013) Slip transmission in bcc FeCr polycrystal. Mater Sci Eng A 588:308 317. doi:10.1016/j.msea.2013.08.050. http://www.sciencedirect. com/science/article/pii/S0921509313009374

18. Kammers A, Daly S (2013) Self-assembled nanoparticle surface patterning for improved digital image correlation in a scanning electron microscope. Exp Mech 53(8):1333-1341. doi:10.1007/s11340-013-9734-5

19. Abuzaid WZ, Sangid MD, Carroll JD, Sehitoglu H, Lambros J (2012) Slip transfer and plastic strain accumulation across grain boundaries in Hastelloy X. J Mech Phys Solids 60(6):1201-1220. doi:10.1016/j.jmps.2012.02.001. http://www. sciencedirect.com/science/article/pii/S0022509612000324

20. Tatschl A, Kolednik O (2003) A new tool for the experimental characterization of micro-plasticity. Mater Sci Eng A 339(1-2):265-280. doi:10.1016/S0921-5093(02)00111-9. http:// www.sciencedirect.com/science/article/pii/S0921509302001119

21. Sutton MA, Li N, Joy DC, Reynolds AP, Li X (2007) Scanning electron microscopy for quantitative small and large deformation measurements Part I: SEM imaging at magnifications from 200 to 10,000. Exp Mech 47(6):775-787. doi:10.1007/s11340-007-9042-z

22. Sutton MA, Li N, Garcia D, Cornille N, Orteu JJ, McNeill SR, Schreier HW, Li X, Reynolds AP (2007) Scanning electron microscopy for quantitative small and large deformation measurements Part II: experimental validation for magnifications from 200 to 10,000. Exp Mech 47(6):789-804. doi:10.1007/s11340-007-9041-0

23. Krueger D, Kissinger R, Menzies R (1992) Superalloys. In: Antolovich SD (ed). TMS-AIME, Warrendale, pp 277-286

24. Miao J, Pollock T, Jones J. (TMS, Warrendale, PA, 2008), pp. 589-597

25. Reed R (2006) The superalloys: fundamentals and applications. Cambridge University Press. http://books.google.com/ books?id=SIUGcd4a-EkC 
26. Sutton MA (2008) Springer handbook of experimental solid mechanics. In: Sharpe J, William N (eds). Springer, US, pp 565600. doi:10.1007/978-0-387-30877-7_20

27. Bridier F, Stinville JC, Vanderesse N, Villechaise P, Bocher $P$ (2014) Microscopic strain and crystal rotation measurement within metallurgical grains. Key Eng Mater 592:493-496

28. Vic-2D (2009) [software] (Correlated Solutions Inc., Columbia, SC)

29. Pan B, Qian K, Xie H, Asundi A (2009) Two-dimensional digital image correlation for in-plane displacement and strain measurement: a review. Meas Sci Technol 20(6):062001. http://stacks.iop. org $/ 0957-0233 / 20 / \mathrm{i}=6 / \mathrm{a}=062001$

30. Sutton M, Orteu J, Schreier H (2009) Image correlation for shape, motion and deformation measurements: basic concepts,theory and applications. Springer. https://books.google. com/books?id=AlkqMxpQMLsC

31. Sutton M, Mingqi C, Peters W, Chao Y, McNeill S (1986) Application of an optimized digital correlation method to planar deformation analysis. Image Vis Comput 4(3):143-150. doi:10.1016/0262-8856(86)90057-0. http://www.sciencedirect. com/science/article/pii/0262885686900570

32. Vendroux G, Knauss WG (1998) Submicron deformation field measurements: Part 2. Improved digital image correlation. Exp Mech 38(2):86-92. doi:10.1007/BF02321649

33. Knauss WG, Chasiotis I, Huang Y (2003) Mechanical measurements at the micron and nanometer scales. Mech Mater 35(36):217-231. doi:10.1016/S0167-6636(02)00271-5. http://www. sciencedirect.com/science/article/pii/S0167663602002715

34. Sutton MA, Li N, Garcia D, Cornille N, Orteu JJ, McNeill SR, Schreier HW, Li X (2006) Metrology in a scanning electron microscope: theoretical developments and experimental validation. Meas Sci Technol 17(10):2613. http://stacks.iop.org/ 0957-0233/17/i=10/a=012
35. Carroll J, Abuzaid W, Lambros J, Sehitoglu H (2010) An experimental methodology to relate local strain to microstructural texture. Rev Sci Instrum 81(8):083703. doi:10.1063/1.3474902. http://scitation.aip.org/content/aip/journal/rsi/81/8/10.1063/1.347 4902

36. Darrell T, Wohn K (1988). In: Proceedings CVPR '88, Computer Society Conference on Computer Vision and Pattern Recognition, 1988, pp 504-509. doi:10.1109/CVPR.1988.196282

37. Carter JL, Uchic MD, Mills MJ (2015) Impact of speckle pattern parameters on DIC strain resolution calculated from in-situ SEM experiments. Springer International Publishing, pp 119126

38. Villechaise P, Cormier J, Billot T, Mendez J (2012). In: 12th International Symposium on Superalloys, pp 15-24

39. Stinville JC, Lenthe WC, Miao J, Pollock TM (2015) A combined grain scale elastic-plastic criterion for identification of fatigue crack initiation sites in a twin containing polycrystalline nickel-base superalloy. Acta Mater (under review)

40. Stinville JC, Bridier F, Bocher P, Pollock TM (2015) High resolution mapping of strain localization near twin boundaries in a nickel-based superalloy. Acta Mater 98:29 42. doi:10.1016/j.actamat.2015.07.016. http://www.sciencedirect. com/science/article/pii/S1359645415004784

41. Heinz A, Neumann P (1990) Crack initiation during high cycle fatigue of an austenitic steel. Acta Metall Mater 38(10):1933-1940. doi:10.1016/0956-7151(90)90305-Z. http:// www.sciencedirect.com/science/article/pii/095671519090305Z

42. Echlin MP, Lenthe WC, Pollock TM (2014) Three-dimensional sampling of material structure for property modeling and design. Integrating Materials and Manufacturing Innovation 3(1):21. doi:10.1186/s40192-014-0021-9 